\title{
An EKF for Lie Groups with Application to Crane Load Dynamics
}

\author{
A. M. Sjøberg ${ }^{1}$ O. Egeland ${ }^{1}$ \\ ${ }^{1}$ MTP, NTNU,N-7052 Trondheim, Norway.E-mail: \{alexander.m.sjoberg, olav.egeland\}@ntnu.no
}

\begin{abstract}
An extended Kalman filter (EKF) for systems with configuration given by matrix Lie groups is presented. The error dynamics are given by the logarithm of the Lie group and are based on the kinematic differential equation of the logarithm, which is given in terms of the Jacobian of the Lie group. The probability distribution is also described in terms of the logarithm as a concentrated Gaussian distribution that is a tightly focused distribution around the identity of the Lie group. The filter is applied to estimation on $S O(3)$ a case where a stereo camera setup tracks a crane wire with a payload. The wire, which is under tension and forms a line is monitored by two 2D-cameras, and a line detector is used to obtain a description of how the wire is projected onto each image plane. A model of a spherical pendulum is applied and the estimator is validated by applying it on simulated data, as well as experimental data.
\end{abstract}

Keywords: Line reconstruction, stereo vision, Lie groups, extended Kalman filter, pendulum

\section{Introduction}

Attitude estimation with Kalman filters is a well-established topic that has received extensive attention. An important contribution was the multiplicative extended Kalman filter (EKF) based on unit quaternions (Lefferts et al., 1982). In this filter, the error dynamics were described by the unit quaternion using the kinematic differential equations of the error quaternion. The update in the filter was done multiplicative so that the result was a unit quaternion. An alternative solution was presented in (Pittelkau, 2003) where the error dynamics were formulated in terms of the rotation vector. A solution with an unscented Kalman filter was presented in Crassidis and Markley (2003). A survey of nonlinear attitude estimation methods that developed from this idea is found in (Crassidis et al., 2007). These methods have been extended to the estimation of position and orientation where dual quaternions have been used in multiplicative filters (Filipe et al., 2015).

A recent development is that extended Kalman filters have been formulated on matrix Lie groups, where the estimation error has been described in terms of the logarithm of the Lie group. This is based on a description where a concentrated Gaussian probability distribution was formulated in terms of the logarithm (Chirikjian, 2012). This was initially introduced and formulated in terms of Euclidean groups (Wang and Chirikjian, 2006), and later also formulated for Bayesian distributions on Lie groups in Wolfe et al. (2011), such that the Gaussian distribution was a function depending on the Lie group states. This description of the probability distribution was further developed for $S E(3)$ by Barfoot and Furgale (2014), who suggested to define the Gaussian distribution based on the Lie algebraic error element instead of the logarithm of the state directly, which can lead to simplification of computation. This was used in (Loianno et al., 2016) for an unscented Kalman filter on $S E(3)$, and in (Brossard et al., 2017) for unscented Kalman filtering of matrix Lie groups. Bourmaud et al. (2013) proposed an extended Kalman filter formulation called D-EKFLG for matrix Lie groups based on the assumption of a concentrated Gaussian distribution. The error dynamics, which are needed in the EKF for covariance computations, were formulated in terms of the error logarithm. The derivation of the error dynamics relied on the Baker-Campbell- 
Hausdorff $(\mathrm{BCH})$ formula, and involved time differentiation of the Lie group elements, and was quite involved. This was also investigated in (Cesic et al., 2017), where details of the development of the error dynamics of the DEKF-LG filter were further investigated. The matrix Lie groups that were used in Bourmaud et al. $(2013,2015)$ and Cesic et al. (2017) included both configuration and velocity components. In the case of $S O(3)$ the resulting matrix Lie group was $S O(3) \times \mathbb{R}^{3}$, where also the angular velocity was included in the group.

Tracking of the pendulum motion of a crane load has been addressed by a number of authors (Rauscher et al., 2018; Inukai and Yoshida, 2012; Kim et al., 2004). In this paper, a stereo camera arrangement is used to track the angle of the load wire. This is done to validate the proposed EKF solution, and also as a potentially viable industrial solution for crane control. Inukai and Yoshida (2012) tracked a single point on the payload using servo-aided stereo vision. Bisgaard et al. (2007) used a single camera to track the position of a payload relative to the body frame. The camera was mounted on an aerial vehicle with a slung load attached. The system configuration was a top-down view to detect a circular white disk for position tracking.

The main contribution of this paper is the formulation of an EKF for matrix Lie groups where the error dynamics is simplified compared to Bourmaud et al. $(2013,2015)$ and Cesic et al. (2017). We do this by using the kinematic differential equation of the logarithm directly in the error dynamics. Moreover, the derivation is based on the differentiation of the exponential mapping instead of a first-order approximation of the $\mathrm{BCH}$ formula, which was used in previous works. The proposed EKF for matrix Lie groups is applied to a problem in $S O(3)$ in simulation and experiments to validate the performance. This includes a comparison of the proposed EKF with the D-EKF-LG method in simulations and for experimental data.

The paper is organized as follows: Section II covers Lie groups and Lie algebras, as well as the structure of probability distribution which is assumed. Section III describes the Extended Kalman filter, while Section IV formulates the EKF for estimation of rotation matrices. Section V and VI describe the geometrical framework and how it is used in the vision part. Section VII provides the spherical pendulum dynamics and how to estimated rotation matrix is utilized in this model. Section VIII presents the findings from simulations, while Section IX presents the experimental results. Section $\mathrm{X}$ discusses the findings from the experiments, and Section XI concludes the paper.

\section{Lie Groups and Lie Algebras}

This section presents the required background for matrix Lie groups based on (Hall, 2003; Faraut, 2008; Chirikjian, 2012). Matrix Lie groups are important in several applica- tions and include the groups $S O(3), S E(2), S E(3)$, and the unit quaternions described by the unit sphere $S(3)$.

\subsection{Exponentials and logarithms}

Consider a matrix Lie group $G \subset G L(n, \mathbb{R})$, where $G L(n, \mathbb{R})$ is the group of all invertible $n \times n$ matrices. Then if $\boldsymbol{X}, \boldsymbol{Y} \in G$ it follows that $\boldsymbol{X} \boldsymbol{Y} \in G$, and there is an inverse $\boldsymbol{X}^{-1} \in G$ so that $\boldsymbol{X} \boldsymbol{X}^{-1}=\boldsymbol{I} \in G$, where $\boldsymbol{I}$ is the identity matrix of the appropriate dimension. Let $\mathfrak{u}$ be an element of the associated Lie algebra $\mathfrak{g} \subset M(n, \mathbb{R})$, where $M(n, \mathbb{R})$ is the algebra of $n \times n$ matrices with real elements. The Lie algebra $\mathfrak{g}$ is the tangent space of $G$ at the identity element. Let $\mathfrak{u} \in \mathfrak{g}$ be the logarithm of $\boldsymbol{X}$, which means that

$$
\boldsymbol{X}=\exp \mathfrak{u}
$$

where the exponential map is defined by

$$
\exp \mathfrak{u} \doteq \sum_{k=0}^{\infty} \frac{\mathfrak{u}^{k}}{k !}
$$

The logarithmic map is the inverse map of the exponential map, and gives

$$
\mathfrak{u}=\log \boldsymbol{X}
$$

The matrix logarithm is given by the series

$$
\log \boldsymbol{X}=\log (\boldsymbol{I}+(\boldsymbol{X}-\boldsymbol{I})) \doteq \sum_{k=1}^{\infty}(-1)^{k+1} \frac{(\boldsymbol{X}-\boldsymbol{I})^{k}}{k}
$$

Let the set $\left\{E_{i}\right\}, i=1 \ldots n$ form an orthonormal basis for the $n$ dimensional Lie algebra $\mathfrak{g}$. An element $\mathfrak{u}$ of a matrix Lie algebra can then be written

$$
\mathfrak{u}=u_{1} E_{1}+\ldots+u_{n} E_{n} \in \mathfrak{g}
$$

where $u_{i}$ are the local coordinates of the Lie algebra. This means that the element $\mathfrak{u}$ can be represented by the vector

$$
\boldsymbol{u}=\left[u_{1}, \ldots, u_{n}\right]^{\mathrm{T}} \in \mathbb{R}^{n}
$$

The conversion between $\mathfrak{u} \in \mathfrak{g}$ and $\boldsymbol{u} \in \mathbb{R}^{n}$ is done following the notation of (Bourmaud et al., 2015) with the operators

$$
[\boldsymbol{u}]_{G}^{\wedge}=\mathfrak{u}, \quad[\mathfrak{u}]_{G}^{\vee}=\boldsymbol{u}
$$

It follows that $\left[[\boldsymbol{u}]_{G}^{\wedge}\right]_{G}^{\vee}=\boldsymbol{u}$ and $\left[[\mathfrak{u}]_{G}^{\vee}\right]_{G}^{\wedge}=\mathfrak{u}$.

\subsection{Adjoint representations}

Let $\mathfrak{b} \in \mathfrak{g}$ be an element of the Lie algebra $\mathfrak{g}$, and let $\boldsymbol{b}=[\mathfrak{b}]_{G}^{\vee}$ be the vector representation. Then the adjoint map $A d_{G}(\boldsymbol{X})$ and its matrix representation $\operatorname{Ad}_{G}(\boldsymbol{X})$ are defined by

$$
\left[\operatorname{Ad}_{G}(\boldsymbol{X}) \boldsymbol{b}\right]_{G}^{\wedge}=A d_{G}(\boldsymbol{X}) \mathfrak{b}=\boldsymbol{X} \mathfrak{b} \boldsymbol{X}^{-1}
$$


Let $\boldsymbol{X}(t)=\exp (t \mathfrak{a})$ where $t \in \mathbb{R}$ and $\mathfrak{a} \in \mathfrak{g}$ is constant. Then the directional derivative of $\boldsymbol{X} \mathfrak{b} \boldsymbol{X}^{-1}$ along $\mathfrak{a}$, where $\mathfrak{b}$ is constant, will be

$$
\left.\frac{\mathrm{d}}{\mathrm{d} t} \exp (t \mathfrak{a}) \mathfrak{b} \exp (-t \mathfrak{a})\right|_{t=0}=\mathfrak{a} \mathfrak{b}-\mathfrak{b} \mathfrak{a} \doteq[\mathfrak{a}, \mathfrak{b}]
$$

where $[\mathfrak{a}, \mathfrak{b}]$ is the Lie bracket. The adjoint $\operatorname{map} a d_{G}(\boldsymbol{a})$ and its matrix form $\operatorname{ad}_{G}(\boldsymbol{a})$ are defined by

$$
\left[\operatorname{ad}_{G}(\boldsymbol{a}) \boldsymbol{b}\right]_{G}^{\wedge}=a d_{G}(\mathfrak{a}) \mathfrak{b}=[\mathfrak{a}, \mathfrak{b}]
$$

where $\boldsymbol{a}=[\mathfrak{a}]_{G}^{\vee}$ and $\boldsymbol{b}=[\mathfrak{b}]_{G}^{\vee}$. It follows that (Klarsfeld and Oteo, 1989)

$$
\left.\left.\left[\operatorname{ad}_{G}(\boldsymbol{a})^{n} \boldsymbol{b}\right]_{G}^{\wedge}=a d_{G}(\mathfrak{a})^{n} \mathfrak{b}=[\underbrace{\mathfrak{a},[\ldots[\mathfrak{a}}_{n}, \mathfrak{b}] \ldots\right]\right]
$$

Let $\boldsymbol{A}, \boldsymbol{X} \in M(n, \mathbb{R})$. The left translation $L_{A}$ and the right translation is defined by $L_{A} \boldsymbol{X}=\boldsymbol{A} \boldsymbol{X}$ and $R_{A} \boldsymbol{X}=$ $\boldsymbol{X} \boldsymbol{A}$. It is noted that

$$
[\mathfrak{a}, \mathfrak{b}]=\left(L_{a}-R_{a}\right) \mathfrak{b}
$$

which gives $R_{a} \mathfrak{b}=L_{a} \mathfrak{b}-[\mathfrak{a}, \mathfrak{b}]$ and

$$
R_{a}^{2} \mathfrak{b}=R_{a}\left(R_{a} \mathfrak{b}\right)=L_{a}^{2} \mathfrak{b}-2 L_{a}[\mathfrak{a}, \mathfrak{b}]+[\mathfrak{a},[\mathfrak{a}, \mathfrak{b}]]
$$

where it is used that $\left[\mathfrak{a}, L_{a} \mathfrak{b}\right]=L_{a}[\mathfrak{a}, \mathfrak{b}]$. This in combination with (10) leads to

$$
R_{u}^{n} \mathfrak{a}=\sum_{i=0}^{n}(-1)^{i}\left(\begin{array}{c}
n \\
i
\end{array}\right) L_{u}^{n-i}\left[\operatorname{ad}_{G}(\boldsymbol{u})^{i} \boldsymbol{a}\right]_{G}^{\wedge}
$$

Moreover, it is noted that

$$
\sum_{j=0}^{\infty} \frac{1}{j !} L_{a}^{j} \mathfrak{b}=\sum_{j=0}^{\infty} \frac{1}{j !} \mathfrak{a}^{j} \mathfrak{b}=\exp (\mathfrak{a}) \mathfrak{b}
$$

\subsection{Right and left Jacobians}

The right and left Jacobians of a Lie group are important to formulate the kinematic differential equations of the logarithm, which will be used in the filter design in this paper. The expressions for the right and left Jacobians will be derived in the following. The derivation is a matrix version of the derivation presented in (Faraut, 2008), and is included here for completeness.

First, it is noted that the body velocity, or right velocity (Chirikjian, 2012), in the Lie group $G$ is given by $\left[\boldsymbol{v}_{r}\right]_{G}^{\wedge}=$ $\boldsymbol{X}^{-1} \dot{\boldsymbol{X}}$, which gives the kinematic differential equation of the Lie Group element in the form

$$
\dot{\boldsymbol{X}}=\boldsymbol{X}\left[\boldsymbol{v}_{r}\right]_{G}^{\wedge}
$$

Next, let $\mathfrak{u}$ be the logarithm of $\boldsymbol{X}$, so that $\boldsymbol{X}=\exp (\mathfrak{u})$, and let $\boldsymbol{u}=[\mathfrak{u}]_{G}^{\vee}$ be the vector form of the logarithm. The time derivative of the exponential function is found from the series expansion of the exponential, which gives

$$
\dot{\boldsymbol{X}}=\left.\frac{\mathrm{d}}{\mathrm{d} t} \exp (\mathfrak{u}+\mathfrak{a} t)\right|_{t=0}=\left.\sum_{k=0}^{\infty} \frac{1}{k !} \frac{\mathrm{d}}{\mathrm{d} t}(\mathfrak{u}+t \mathfrak{a})^{k}\right|_{t=0}
$$

To proceed, it is noted that

$$
\left.\frac{\mathrm{d}}{\mathrm{d} t}(\mathfrak{u}+t \mathfrak{a})^{k}\right|_{t=0}=\sum_{j=0}^{k-1} \mathfrak{u}^{k-j-1} \mathfrak{a u}^{j}=\sum_{j=0}^{k-1} L_{u}^{k-j-1} R_{u}^{j} \mathfrak{a}
$$

Insertion of (13) and

$$
\sum_{j=0}^{k-1}\left(\begin{array}{l}
j \\
i
\end{array}\right)=\left(\begin{array}{c}
k \\
i+1
\end{array}\right)
$$

which is derived in (Faraut, 2008), gives

$$
\begin{aligned}
\dot{\boldsymbol{X}} & =\sum_{k=1}^{\infty} \frac{1}{k !} \sum_{i=0}^{k-1}(-1)^{i}\left(\begin{array}{c}
k \\
i+1
\end{array}\right) L_{u}^{j-i}\left[\operatorname{ad}_{G}(\boldsymbol{u})^{i} \boldsymbol{a}\right]_{G}^{\wedge} \\
& =\sum_{j=0}^{\infty} \frac{1}{j !} L_{u}^{j} \sum_{i=0}^{\infty} \frac{(-1)^{i}}{(i+1) !}\left[\operatorname{ad}_{G}(\boldsymbol{u})^{i} \boldsymbol{a}\right]_{G}^{\wedge}
\end{aligned}
$$

It follows from (14) that

$$
\dot{\boldsymbol{X}}=\boldsymbol{X}\left[\boldsymbol{\Psi}_{r}(\operatorname{ad}(\boldsymbol{u})) \dot{\boldsymbol{u}}\right]_{G}^{\wedge}
$$

where $\boldsymbol{\Psi}_{r}(\operatorname{ad}(\boldsymbol{u}))$ is the right Jacobian defined by

$$
\boldsymbol{\Psi}_{r}(\mathbf{a d}(\boldsymbol{u}))=\sum_{n=0}^{\infty} \frac{(-\operatorname{ad}(\boldsymbol{u}))^{i}}{(i+1) !}
$$

The inverse of the right Jacobian is given by

$$
\boldsymbol{\Psi}_{r}^{-1}(\mathbf{a d}(\boldsymbol{u}))=\sum_{i=0}^{\infty} \frac{B_{i}(-\mathbf{a d}(\boldsymbol{u}))^{i}}{i !}
$$

where $B_{n}$ are the Bernoulli numbers given by $B_{0}=1$, $B_{1}=-\frac{1}{2}, B_{2}=\frac{1}{6}, B_{3}=0, B_{4}=-\frac{1}{30}, B_{5}=0, \ldots$ Closed-form solutions for the right Jacobian and its inverse are given in (Park, 1995) for $S O(3)$,

From (15) and (19) it is seen that $\boldsymbol{v}_{r}=\boldsymbol{\Psi}_{r}(\operatorname{ad}(\boldsymbol{u})) \dot{\boldsymbol{u}}$, and it follows that the kinematic differential equation for logarithm is

$$
\dot{\boldsymbol{u}}=\boldsymbol{\Psi}_{r}^{-1}(\operatorname{ad}(\boldsymbol{u})) \boldsymbol{v}_{r}
$$

It is noted that there is also a left Jacobian

$$
\boldsymbol{\Psi}_{l}(\operatorname{ad}(\boldsymbol{u}))=\boldsymbol{\Psi}_{r}(-\operatorname{ad}(\boldsymbol{u}))
$$

which appears in

$$
\dot{\boldsymbol{X}}=\left[\boldsymbol{\Psi}_{l}(\operatorname{ad}(\boldsymbol{u})) \dot{\boldsymbol{u}}\right]_{G}^{\wedge} \boldsymbol{X}
$$


The left Jacobian is found with a derivation that follows the same steps as the derivation of the right Jacobian, with the difference that

$$
L_{u}^{n} \mathfrak{a}=\sum_{i=0}^{n}\left(\begin{array}{c}
n \\
i
\end{array}\right) R_{u}^{n-i}\left[\operatorname{ad}_{G}(\boldsymbol{u})^{i} \boldsymbol{a}\right]_{G}^{\wedge}
$$

is used in place of (13) and

$$
\sum_{j=0}^{\infty} \frac{1}{j !} R_{a}^{j} \mathfrak{b}=\sum_{j=0}^{\infty} \mathfrak{b} \frac{1}{j !} \mathfrak{a}^{j}=\mathfrak{b} \exp (\mathfrak{a})
$$

is used in place of (14). The use of the left Jacobian gives the alternative kinematic differential equation

$$
\dot{\boldsymbol{u}}=\boldsymbol{\Psi}_{l}^{-1}(\operatorname{ad}(\boldsymbol{u})) \boldsymbol{v}_{l}
$$

of the logarithm in terms of the spatial, or left, velocity $\left[\boldsymbol{v}_{l}\right]_{G}^{\wedge}=\mathfrak{v}_{l}=\dot{\boldsymbol{X}} \boldsymbol{X}^{-1}$.

Closed-form solutions for the left and right Jacobians and their inverses were presented for $S O(3)$ in (Park, 1995), while a closed-form solution for the left Jacobian of $S E(3)$ was presented in (Barfoot and Furgale, 2014).

\subsection{Baker-Campbell-Hausdorff Formula}

Let $\mathfrak{a}=[\boldsymbol{a}]_{G}^{\wedge}, \mathfrak{b}=[\boldsymbol{b}]_{G}^{\wedge}$ and $\mathfrak{c}=[\boldsymbol{c}]_{G}^{\wedge}$ be elements of the Lie algebra $\mathfrak{g}$, and suppose that

$$
\exp (\mathfrak{c})=\exp (\mathfrak{a}) \exp (\mathfrak{b})
$$

Then the Baker-Campbell-Hausdorff (BHC) formula gives (Hall, 2003)

$$
\mathfrak{c}=\mathfrak{a}+\mathfrak{b}+\frac{1}{2}[\mathfrak{a}, \mathfrak{b}]+\frac{1}{12}([\mathfrak{a},[\mathfrak{a}, \mathfrak{b}]]+[\mathfrak{b},[\mathfrak{b}, \mathfrak{a}])+\ldots
$$

If only linear terms in $\mathfrak{b}$ are included, then the $\mathrm{BCH}$ formula can be approximated by (Klarsfeld and Oteo, 1989)

$$
\boldsymbol{c} \approx \boldsymbol{a}+\boldsymbol{\Psi}_{r}^{-1}[\operatorname{ad}(\boldsymbol{a})] \boldsymbol{b}
$$

where $\boldsymbol{\Psi}_{r}$ is the right Jacobian defined in (20).

\subsection{Discrete-time kinematic differential equation}

The time propagation of $\boldsymbol{X}(t)$ from time $t_{k}$ to time $t_{k+1}=$ $t_{k}+T$ can be found by applying Euler's method to the kinematic differential equation (15), which gives

$$
\boldsymbol{X}\left(t_{k+1}\right)=\boldsymbol{X}\left(t_{k}\right) \exp \left(T \mathfrak{v}_{b}\left(t_{k}\right)\right)
$$

In terms of the logarithm $\mathfrak{u}=\log (\boldsymbol{X})$, this is written

$$
\exp \left[\mathfrak{u}\left(t_{k+1}\right)\right]=\exp \left[\mathfrak{u}\left(t_{k}\right)\right] \exp \left[T \mathfrak{v}_{b}\left(t_{k}\right)\right]
$$

Alternatively, Euler's method can be applied to the kinematic differential equation (27) of the logarithm (Iserles et al., 2005), which gives

$$
\boldsymbol{u}\left(t_{k+1}\right)=\boldsymbol{u}\left(t_{k}\right)+T \boldsymbol{\Psi}_{r}^{-1}\left[\operatorname{ad}\left(\boldsymbol{u}\left(t_{k}\right)\right)\right] \boldsymbol{v}_{r}\left(t_{k}\right)
$$

where $\boldsymbol{v}_{r}=\left[\mathfrak{v}_{b}\right]_{G}^{\vee}$. Note that this result can also be found by applying the approximation of the $\mathrm{BCH}$ formula (29) to (31).

The Crouch-Grossman method for time integration of dynamics on Lie groups was developed for the kinematic differential equation (19) or (24) of the Lie group element $\boldsymbol{X}$ using the exponential functions (Crouch and Grossman, 1993; Owren and Marthinsen, 1999). A more recent development is the RK-MK method where the kinematic differential equation for the logarithm (22) or (27) is used to design Runge-Kutta methods for Lie groups (Munthe-Kaas, 1995; Iserles et al., 2005).

\subsection{Concentrated Gaussian Distribution}

Estimation of elements describing the state of the system requires a description of the uncertainties of the system, and filters based on the Kalman filter assumes that the probability distributions associated with the states are Gaussian. In the case where the system state resides on a matrix Lie group $G$, the Gaussian distribution in Euclidean space may not be sufficient as the inherent properties may be different.

In Wang and Chirikjian (2006) the distribution

$$
p(\boldsymbol{X}) \sim \alpha \exp _{G}\left(-\frac{1}{2} \boldsymbol{\epsilon}^{\mathrm{T}} \boldsymbol{P}^{-1} \boldsymbol{\epsilon}\right)
$$

was introduced as a Gaussian distribution where $\boldsymbol{X}=$ $\exp \left([\boldsymbol{\epsilon}]_{G}^{\wedge}\right)$ resides on the Lie group $G,[\boldsymbol{\epsilon}]_{G}^{\wedge}$ is the logarithm of $\boldsymbol{X}$, and $\boldsymbol{P}$ is the covariance matrix of the system. The scalar $\alpha$ normalizes the distribution such that the integral of the Gaussian distribution $p$ evaluates to 1 .

If the Gaussian distribution $p$ is tightly focused around the identity of the Lie group, we call it a concentrated Gaussian distribution (CGD). Assuming a CGD makes it possible to utilize Gaussian distributions defined in the Euclidean space as local approximations of Gaussians residing on manifolds of Lie groups. The requirement is that the Lie group of interest to be a unimodular connected (Hall, 2003) matrix Lie group (Wolfe et al., 2011). A unimodular Lie group is a set of elements which possesses a biinvariant Haar measure, which is the case for the Lie groups $S O(3)$ and $S E(3)$ (Chirikjian, 2012). Connectedness in a Lie group $G$ is achieved if for any two matrices, $\boldsymbol{A}, \boldsymbol{B} \in G$, there is a continuous path $\boldsymbol{A}(t) \in G$ parameterized by a scalar $t \in[a, b]$, with $\boldsymbol{A}(a)=\boldsymbol{A}$ and $\boldsymbol{A}(b)=\boldsymbol{B}$. The matrix Lie group $S O(3)$ is an example of a unimodular connected matrix Lie group. Under the assumption that the uncertainties are CGD, we can use the distribution

$$
\boldsymbol{\epsilon}=\left[\log _{G} \boldsymbol{X}\right]_{G}^{\vee} \sim \mathcal{N}_{\mathbb{R}^{6}}\left(\mathbf{0}_{6 \times 1}, \boldsymbol{P}_{6 \times 6}\right)
$$


as a valid approximation of the Gaussian distribution on the manifold in the identity. The corresponding distribution on the Lie group is

$$
\exp _{G}(\boldsymbol{\epsilon}) \sim \mathcal{N}_{G}\left(\boldsymbol{I}, \boldsymbol{P}_{6 \times 6}\right)
$$

which is illustrated in Figure (1). Furthermore, if the assumption of CGD holds, any distribution of $\boldsymbol{X}$ on $G$ can be approximated as

$$
\boldsymbol{X}=\boldsymbol{\mu} \exp _{G}(\boldsymbol{\epsilon}) \sim \mathcal{N}_{G}\left(\boldsymbol{\mu}, \boldsymbol{P}_{6 \times 6}\right)
$$

which means that distribution on $G$ in the identity can be transported from the distribution at the identity to the mean value $\boldsymbol{\mu} \in G$ by using the left action on the Lie group. This is illustrated in Figure (2).

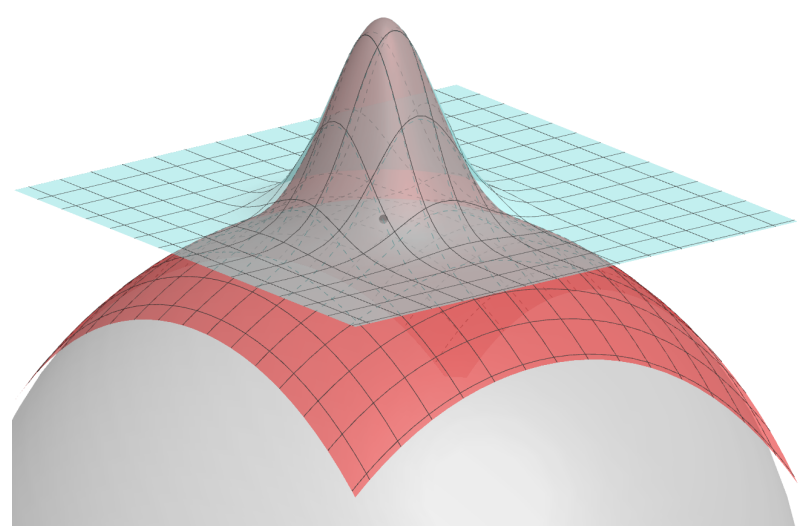

Figure 1: The logarithm of $\boldsymbol{X}$ at the origin of the Lie algebra (blue surface) approximates the Gaussian distribution at the group identity (red surface) if the distribution is tightly focused near the identity.

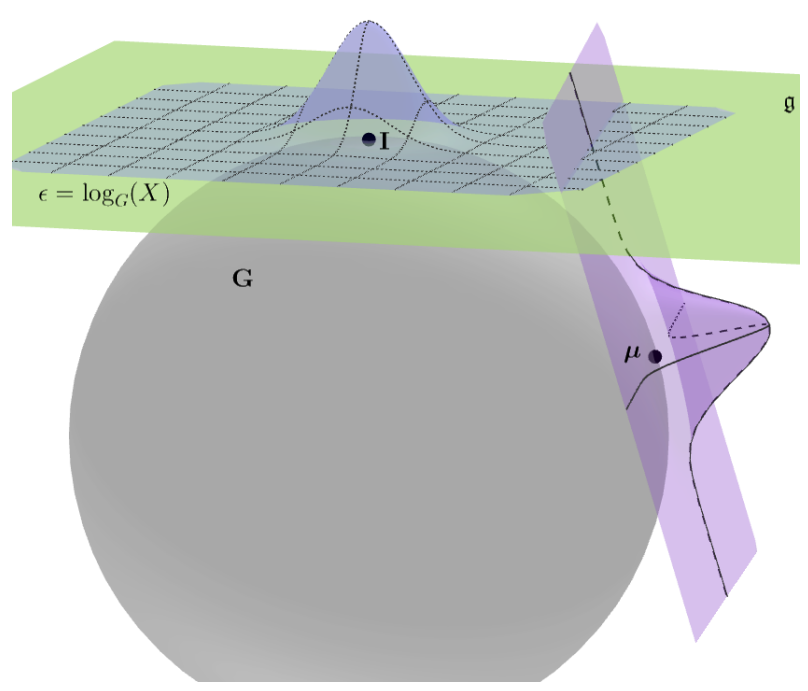

Figure 2: The Gaussian distribution of $\boldsymbol{X}$ on the mean element $\boldsymbol{\mu}$ is obtained by transporting the approximated Gaussian distribution at the identity of $G$ to the mean state $\boldsymbol{\mu}$.

\section{Extended Kalman Filter}

\subsection{State Representation}

Let the state be given by

$$
\boldsymbol{x}=\left\{\boldsymbol{X}, \boldsymbol{v}_{r}\right\} \in G \times \mathbb{R}^{n}
$$

where $\boldsymbol{X} \in G$ and $\left[\boldsymbol{v}_{r}\right]_{G}^{\wedge}=\boldsymbol{X}^{-1} \dot{\boldsymbol{X}} \in \mathbb{R}^{n \times n}$ is the body velocity in $G$. The dynamics of the state are given by

$$
\begin{aligned}
\dot{\boldsymbol{X}} & =\boldsymbol{X}\left[\boldsymbol{v}_{r}\right]_{G}^{\wedge} \\
\dot{\boldsymbol{v}}_{r} & =\boldsymbol{f}_{v}(\boldsymbol{x})
\end{aligned}
$$

The design of the EKF is based on the estimation error $\delta \boldsymbol{X}=\overline{\boldsymbol{X}}^{-1} \boldsymbol{X}$ where $\boldsymbol{X}$ is the actual variable and $\overline{\boldsymbol{X}}$ is the estimated variable. Let $[\boldsymbol{\epsilon}]_{G}^{\wedge}=\log (\delta \boldsymbol{X})$ be the logarithm of the estimation error, so that

$$
\delta \boldsymbol{X}=\exp \left([\boldsymbol{\epsilon}]_{G}^{\wedge}\right)
$$

which gives

$$
\boldsymbol{X}=\overline{\boldsymbol{X}} \delta \boldsymbol{X}=\overline{\boldsymbol{X}} \exp \left([\boldsymbol{\epsilon}]_{G}^{\wedge}\right)
$$

Define the error velocity by $\left[\delta \boldsymbol{v}_{r}\right]_{G}^{\wedge}=\delta \boldsymbol{X}^{-1} \delta \dot{\boldsymbol{X}} \in \mathbb{R}^{n \times n}$. Then

$$
\begin{aligned}
\delta \dot{\boldsymbol{X}} & =\delta \boldsymbol{X}\left[\delta \boldsymbol{v}_{r}\right]_{G}^{\wedge} \\
\delta \dot{\boldsymbol{v}}_{r} & =\boldsymbol{f}_{v e}(\boldsymbol{x})
\end{aligned}
$$

Then it follows from (22) that the kinematic differential equation of the logarithm is

$$
\dot{\boldsymbol{\epsilon}}=\boldsymbol{\Psi}_{R}^{-1}(\operatorname{ad}(\boldsymbol{\epsilon})) \delta \boldsymbol{v}_{r}
$$

where $\boldsymbol{\Psi}_{r}(\operatorname{ad}(\boldsymbol{\epsilon}))$ is the right Jacobian defined in (20).

\subsection{Time propagation of EKF}

The time propagation of the estimate $\overline{\boldsymbol{x}}=\left\{\overline{\boldsymbol{X}}, \overline{\boldsymbol{v}}_{r}\right\}$ is done by time integration of the system dynamics $(38,39)$. The predicted states are computed from

$$
\begin{aligned}
\boldsymbol{X}_{k+1 \mid k} & =\boldsymbol{X}_{k \mid k} \exp \left(T \hat{\boldsymbol{v}}_{r, k \mid k}+\frac{1}{2} T^{2}\left[\boldsymbol{f}_{v}\left(\boldsymbol{x}_{k \mid k}\right)\right]_{G}^{\wedge}\right) \\
\boldsymbol{v}_{r, k+1 \mid k} & =\boldsymbol{v}_{r, k \mid k}+T \boldsymbol{f}_{v}\left(\boldsymbol{x}_{k \mid k}\right)
\end{aligned}
$$

The propagation of the covariance matrix is based on the linearization of the error dynamics about the estimate, hence the covariance propagation is computed from

$$
\boldsymbol{P}_{k+1 \mid k}=\boldsymbol{F}_{k} \boldsymbol{P}_{k \mid k} \boldsymbol{F}_{k}^{\mathrm{T}}+\boldsymbol{R}_{n}
$$

where $\boldsymbol{R}_{n}$ is the covariance matrix describing the process noise. The state propagation matrix $\boldsymbol{F}_{k}$ is derived from the 
error dynamics $\dot{\boldsymbol{x}}_{e}=\boldsymbol{f}\left(\boldsymbol{x}_{e}\right)$. Let $\boldsymbol{x}_{e}=\left[\boldsymbol{\epsilon}^{\mathrm{T}}, \delta \boldsymbol{v}_{b}^{\mathrm{T}}\right]^{\mathrm{T}}$ be the error states defined in the Lie algebra, and let

$$
\boldsymbol{x}_{e, k+1 \mid k}=\left[\begin{array}{c}
\epsilon_{k \mid k}+T \delta \boldsymbol{v}_{b, k \mid k}+\frac{1}{2} T^{2} \boldsymbol{f}_{v e}\left(\boldsymbol{x}_{e, k \mid k}\right) \\
\delta \boldsymbol{v}_{b, k \mid k}+T \boldsymbol{f}_{v e}\left(\boldsymbol{x}_{e, k \mid k}\right)
\end{array}\right]
$$

be the discretized solution based on the dynamics of Equation $(44,43)$. Since the expected mean of $\epsilon$ is given as $\mathbb{E}\{\boldsymbol{\epsilon}\}=\mathbf{0}$, this yields $\boldsymbol{\Psi}_{r}^{-1}(\boldsymbol{\epsilon})=\boldsymbol{I}$. If the process is modeled as only driven by noise with $\boldsymbol{f}_{v e}=\mathbf{0}$, then

$$
\boldsymbol{F}=\left.\frac{\partial \boldsymbol{f}\left(\boldsymbol{x}_{e}\right)}{\partial \boldsymbol{x}_{e}}\right|_{\boldsymbol{x}_{e}=\mathbf{0}}=\left[\begin{array}{cc}
\boldsymbol{I} & T \boldsymbol{I} \\
\mathbf{0} & \boldsymbol{I}
\end{array}\right]
$$

\subsection{Update}

Suppose that the measurements are given in the form of Lie group elements $\boldsymbol{Z}_{k} \in G$. The measurement model is

$$
\boldsymbol{Z}=\boldsymbol{X} \exp \left([\boldsymbol{w}]_{G}^{\wedge}\right)
$$

where $\boldsymbol{w} \sim \mathcal{N}\left(\mathbf{0}, Q_{w}\right)$ is white Gaussian noise describing the measurement noise, with covariance matrix $\boldsymbol{Q}_{w}$. We express the difference between the measured and predicted matrix Lie group elements, e. g. a rotation matrix, in terms of the logarithm of the error as

$$
\left.\boldsymbol{y}_{k+1}=\left[\log \left(\overline{\boldsymbol{X}}_{k+1 \mid k}\right)^{-1} \boldsymbol{Z}_{k+1}\right)\right]_{G}^{\vee} \in \mathbb{R}^{3}
$$

The measurement matrix is found as the Jacobian

$$
\boldsymbol{H}=\left[\begin{array}{ll}
\boldsymbol{I}_{3 \times 3} & \mathbf{0}_{3 \times 3}
\end{array}\right]
$$

The Kalman gain is computed as

$$
\boldsymbol{K}_{k+1}=\boldsymbol{P}_{k+1 \mid k} \boldsymbol{H}^{\mathrm{T}}\left(\boldsymbol{H} \boldsymbol{P}_{k+1 \mid k} \boldsymbol{H}^{\mathrm{T}}+\boldsymbol{Q}_{w}\right)^{-1}
$$

As the error between the measurement $\boldsymbol{Z}_{k}$ and the expected measurement $h\left(\boldsymbol{x}_{k+1 \mid k}\right)$ is represented in the Lie algebra, we obtain the correction term

$$
\boldsymbol{m}_{k+1}=\boldsymbol{K}_{k+1} \boldsymbol{y}_{k+1}=\left[\begin{array}{c}
\boldsymbol{m}_{X, k+1} \\
\boldsymbol{m}_{v, k+1}
\end{array}\right] \in \mathbb{R}^{6}
$$

The elements in the state posterior $\boldsymbol{x}_{k+1 \mid k+1}$ are then computed as

$$
\begin{aligned}
\overline{\boldsymbol{X}}_{k+1 \mid k+1} & =\overline{\boldsymbol{X}}_{k+1 \mid k} \exp \left(\left[\boldsymbol{m}_{X, k+1}\right]_{G}^{\wedge}\right) \\
\boldsymbol{v}_{k+1 \mid k+1} & =\boldsymbol{v}_{k+1 \mid k}+\boldsymbol{m}_{v, k+1}
\end{aligned}
$$

Finally, the posterior covariance is found as

$$
\boldsymbol{P}_{k \mid k}=\left(\boldsymbol{I}-\boldsymbol{K}_{k} \boldsymbol{H}\right) \boldsymbol{P}_{k \mid k-1}
$$

To summarize the EKF, we provide the structure of the estimator in Algorithm (1).

\section{EKF Algorithm:}

Inputs: $\boldsymbol{x}_{k \mid k}, \boldsymbol{P}_{k \mid k}, \boldsymbol{Z}_{k+1}$
Outputs: $\boldsymbol{x}_{k+1 \mid k+1}, \boldsymbol{P}_{k+1 \mid k+1}$
Initialization: $\boldsymbol{x}_{0 \mid 0}=\{\boldsymbol{I}, \mathbf{0}\}, \boldsymbol{P}_{0 \mid 0}=\boldsymbol{I}$

Propagation:

$$
\begin{aligned}
& \boldsymbol{X}_{k+1 \mid k}=\boldsymbol{X}_{k \mid k} \exp \left(T \hat{\boldsymbol{v}}_{k \mid k}+\frac{1}{2} T^{2}\left[\boldsymbol{f}_{v}\left(\boldsymbol{x}_{k \mid k}\right)\right]_{G}^{\wedge}\right) \\
& \boldsymbol{v}_{k+1 \mid k}=\boldsymbol{v}_{k \mid k}+T \boldsymbol{f}_{v}\left(\boldsymbol{x}_{k \mid k}\right) \\
& \boldsymbol{P}_{k+1 \mid k}=\boldsymbol{F}_{k} \boldsymbol{P}_{k \mid k} \boldsymbol{F}_{k}^{\mathrm{T}}+\boldsymbol{R}_{n}
\end{aligned}
$$

\section{Update:}

$$
\begin{aligned}
& \boldsymbol{K}_{k+1}=\boldsymbol{P}_{k+1 \mid k} \boldsymbol{H}^{\mathrm{T}}\left(\boldsymbol{H} \boldsymbol{P}_{k+1 \mid k} \boldsymbol{H}^{\mathrm{T}}+\boldsymbol{Q}_{w}\right)^{-1} \\
& \boldsymbol{m}_{k+1}=\boldsymbol{K}_{k+1} \log \left(\boldsymbol{X}_{k+1 \mid k}^{-1} \boldsymbol{Z}_{k+1}\right) \\
& \quad=\left[\boldsymbol{m}_{R, k+1}, \boldsymbol{m}_{\omega, k+1}\right]^{\mathrm{T}} \\
& \boldsymbol{X}_{k+1 \mid k+1}=\boldsymbol{X}_{k+1 \mid k} \exp \left(\left[\boldsymbol{m}_{R, k+1}\right]_{G}^{\wedge}\right) \\
& \boldsymbol{v}_{k+1 \mid k+1}=\boldsymbol{v}_{k+1 \mid k}+\boldsymbol{m}_{\omega, k+1} \\
& \boldsymbol{P}_{k+1 \mid k+1}=\left(\boldsymbol{I}-\boldsymbol{K}_{k+1} \boldsymbol{H}\right) \boldsymbol{P}_{k+1 \mid k}
\end{aligned}
$$

Algorithm 1: The structure of the EKF.

\section{Extended Kalman Filter on $S O(3)$}

A rotation matrix $\boldsymbol{R}$ is an element of the matrix Lie group $S O(3)$ defined by

$$
S O(3) \doteq\left\{\boldsymbol{R} \in \mathbb{R}^{3 \times 3} \mid \boldsymbol{R} \boldsymbol{R}^{\mathrm{T}}=\boldsymbol{I}_{3 \times 3}, \operatorname{det} \boldsymbol{R}=1\right\}
$$

where the inverse is $\boldsymbol{R}^{-1}=\boldsymbol{R}^{\mathrm{T}}$. The corresponding Lie algebra is $\mathfrak{s o}(3)$, with elements

$$
\mathfrak{u}=[\boldsymbol{u}]_{S O(3)}^{\wedge}=\left[\begin{array}{ccc}
0 & -u_{z} & u_{y} \\
u_{z} & 0 & -u_{x} \\
-u_{y} & u_{x} & 0
\end{array}\right] \in \mathfrak{s o}(3)
$$

which is the skew-symmetric form of the vector $\boldsymbol{u}=$ $\left[u_{x}, u_{y}, u_{z}\right]^{\mathrm{T}} \in \mathbb{R}^{3}$. The notation $\hat{\boldsymbol{u}}=[\boldsymbol{u}]_{S O(3)}^{\wedge}$ will also be used. It is noted that $\hat{\boldsymbol{u}} \boldsymbol{w}=\boldsymbol{u} \times \boldsymbol{w}$ for $\boldsymbol{u}, \boldsymbol{w} \in \mathbb{R}^{3}$. It is well-known that the skew-symmetric form $\hat{\boldsymbol{u}}$ of a vector $\boldsymbol{u}$ transforms in $S O(3)$ according to $[\boldsymbol{R u}]_{S O(3)}^{\wedge}=\boldsymbol{R} \hat{\boldsymbol{u}} \boldsymbol{R}^{\mathrm{T}}$. Comparison with (7) shows that

$$
\operatorname{Ad}_{S O(3)}(\boldsymbol{R})=\boldsymbol{R}
$$

From linear algebra it is known that the skew-symmetric form of the vector $(\hat{\boldsymbol{u}} \boldsymbol{w})$ is $(\hat{\boldsymbol{u}} \boldsymbol{w})^{\wedge}=\hat{\boldsymbol{u}} \hat{\boldsymbol{w}}-\hat{\boldsymbol{w}} \hat{\boldsymbol{u}}$, and comparison with (9) gives

$$
\operatorname{ad}_{S O(3)}(\boldsymbol{u})=\hat{\boldsymbol{u}}
$$


The time derivative of the rotation matrix is given by $\dot{\boldsymbol{R}}=$ $\boldsymbol{R} \hat{\boldsymbol{\omega}}$, where $\hat{\boldsymbol{\omega}}$ is the skew-symmetric form of the angular velocity vector $\boldsymbol{\omega}$. The time derivative at $\boldsymbol{R}=\boldsymbol{I}$ is therefore $\left.\dot{\boldsymbol{R}}\right|_{\boldsymbol{R}=\boldsymbol{I}}=\hat{\boldsymbol{\omega}}$. The matrix exponential and the logarithm are given by

$$
\boldsymbol{R}=\exp _{S O(3)} \mathfrak{u}, \quad \mathfrak{u}=\log (\boldsymbol{R})
$$

As pointed out in (Bullo and Murray, 1995), the Lie algebra element $\mathfrak{u} \in \mathfrak{s o}(3)$ is a local parameterization of the rotation matrix $\boldsymbol{R}$, while the Lie algebra element $\hat{\boldsymbol{\omega}} \in \mathfrak{s o}(3)$ is related to the time derivative of the Lie Group element $\boldsymbol{R}$.

Consider the case where the logarithm is given by $\mathfrak{u}=\theta \hat{\boldsymbol{k}}$ where $\boldsymbol{k} \in \mathbb{R}^{3}$ is a unit vector. Then $\boldsymbol{R}$ is a rotation by an angle of $\theta$ about $\boldsymbol{k}$, and is given by the Rodrigues equation

$$
\boldsymbol{R}=\boldsymbol{I}+\sin \theta \hat{\boldsymbol{k}}+(1-\cos \theta) \hat{\boldsymbol{k}} \hat{\boldsymbol{k}}
$$

The $\operatorname{logarithm}$ is $\log (\boldsymbol{R})=\theta \hat{\boldsymbol{k}}$, which can be computed from (Iserles et al., 2005)

$$
\log (\boldsymbol{R})=\frac{\arcsin (\|\boldsymbol{w}\|)}{\|\boldsymbol{w}\|} \hat{\boldsymbol{w}}, \quad \hat{\boldsymbol{w}}=\frac{1}{2}\left(\boldsymbol{R}-\boldsymbol{R}^{\mathrm{T}}\right)
$$

The right Jacobian and its inverse as given by equations (20) and (21), have the following closed-form solutions in $S O(3)$ (Bullo and Murray, 1995; Chirikjian, 2012)

$$
\begin{gathered}
\Psi_{r}(\hat{\boldsymbol{u}})=\boldsymbol{I}-\frac{1-\cos \|\boldsymbol{u}\|}{\|\boldsymbol{u}\|^{2}} \hat{\boldsymbol{u}}+\frac{\|\boldsymbol{u}\|-\sin \|\boldsymbol{u}\|}{\|\boldsymbol{u}\|^{3}} \hat{\boldsymbol{u}}^{2} \\
\boldsymbol{\Psi}_{r}^{-1}(\hat{\boldsymbol{u}})=\boldsymbol{I}+\frac{1}{2} \hat{\boldsymbol{u}}+\left(\frac{1-\frac{\|\boldsymbol{u}\|}{2} \cot \frac{\|\boldsymbol{u}\|}{2}}{\|\boldsymbol{u}\|^{2}}\right) \hat{\boldsymbol{u}}^{2}
\end{gathered}
$$

The Taylor series expansion of the last term is found from $\cot x=x^{-1}-\frac{1}{3} x+\frac{1}{45} x^{3}-\frac{2}{945} x^{5}-\ldots$ to be

$$
\frac{1-\frac{\theta}{2} \cot \frac{\theta}{2}}{4\left(\frac{\theta}{2}\right)^{2}}=\frac{1}{12}+\frac{1}{180}\left(\frac{\theta}{2}\right)^{2}+\frac{1}{1890}\left(\frac{\theta}{2}\right)^{4}+\ldots
$$
then

The kinematic differential equation for the logarithm is

$$
\dot{\hat{\boldsymbol{u}}}=\boldsymbol{\Psi}_{r}^{-1}(\hat{\boldsymbol{u}}) \hat{\boldsymbol{\omega}}
$$

The equation was also used in (Bortz, 1970) and in (Pittelkau, 2003), where the approximation

$$
\Psi_{r}^{-1}(\hat{\boldsymbol{u}}) \approx \boldsymbol{I}+\frac{1}{2} \hat{\boldsymbol{u}}+\frac{1}{12} \hat{\boldsymbol{u}}^{2}
$$

was recommended for the kinematic differential equation (68), which means that the first term in the Taylor series expansion (67) is used.

\subsection{State Representation}

Let the element

$$
\boldsymbol{x}=\{\boldsymbol{R}, \boldsymbol{\omega}\} \in S O(3) \times \mathbb{R}^{3}
$$

represent the system state, where $\boldsymbol{R} \in S O(3)$ describes the attitude and $\boldsymbol{\omega} \in \mathbb{R}^{3}$ describes the angular velocity. The state dynamics, in continuous form, is

$$
\dot{\boldsymbol{x}}=\{\dot{\boldsymbol{R}}, \dot{\boldsymbol{\omega}}\}
$$

where the dynamics are explicitly given as the following kinematic differential equations

$$
\begin{aligned}
\dot{\boldsymbol{R}} & =\boldsymbol{R} \hat{\boldsymbol{\omega}} \\
\dot{\boldsymbol{\omega}} & =\boldsymbol{f}_{\omega}(\boldsymbol{x})
\end{aligned}
$$

and $\boldsymbol{\omega}^{b}$ is the angular velocity defined in the body frame.

Let $s$ be the spatial frame, $e$ be the estimated body frame, and let $b$ be the body frame. Then the actual rotation $\boldsymbol{R}=\boldsymbol{R}_{b}^{s}$ is the rotation matrix from $s$ to $b$, the estimated rotation $\hat{\boldsymbol{R}}=\boldsymbol{R}_{e}^{s}$ is the rotation matrix from $s$ to $e$, and the estimation error

$$
\delta \boldsymbol{R}=\hat{\boldsymbol{R}}^{\mathrm{T}} \boldsymbol{R}=\boldsymbol{R}_{b}^{e}
$$

is the rotation matrix from $e$ to $b$. The kinematic differential equations are then $\dot{\boldsymbol{R}}_{b}^{s}=\boldsymbol{R}_{b}^{s} \hat{\boldsymbol{\omega}}_{s b}^{b}, \hat{\boldsymbol{R}}_{e}^{s}=\hat{\boldsymbol{R}}_{e}^{s} \hat{\boldsymbol{\omega}}_{s e}^{e}$ and

$$
\dot{\boldsymbol{R}}_{b}^{e}=\boldsymbol{R}_{b}^{e} \hat{\boldsymbol{\omega}}_{e b}^{b}
$$

where $\boldsymbol{\omega}_{e b}^{b}=\boldsymbol{\omega}_{i b}^{b}-\boldsymbol{\omega}_{i e}^{b}$.

Let $[\boldsymbol{\epsilon}]_{S O(3)}^{\wedge}=\log (\delta \boldsymbol{R})$ be the logarithm of the error rotation. Then the kinematic differential equation for the vector form $\epsilon$ of the logarithm is found from (22) to be

$$
\dot{\boldsymbol{\epsilon}}=\boldsymbol{\Psi}_{R}^{-1}(\operatorname{ad}(\boldsymbol{\epsilon})) \boldsymbol{\omega}_{e b}^{b}
$$

\subsection{Time propagation of EKF}

The predicted states are computed from

$$
\begin{aligned}
\boldsymbol{R}_{k+1 \mid k} & =\boldsymbol{R}_{k \mid k} \exp \left(T \hat{\boldsymbol{\omega}}_{k \mid k}+\frac{1}{2} T^{2}\left[\boldsymbol{f}_{\omega}\left(\boldsymbol{x}_{k}\right)\right]_{S O(3)}^{\wedge}\right) \\
\boldsymbol{\omega}_{k+1 \mid k} & =\boldsymbol{\omega}_{k \mid k}+T \boldsymbol{f}_{\omega}\left(\boldsymbol{x}_{k}\right)
\end{aligned}
$$

The covariance propagation is given by

$$
\boldsymbol{P}_{k \mid k-1}=\boldsymbol{F}_{k-1} \boldsymbol{P}_{k-1 \mid k-1} \boldsymbol{F}_{k-1}^{\mathrm{T}}+\boldsymbol{R}_{n}
$$

where $\boldsymbol{R}_{n}$ is the covariance matrix describing the process noise, and $\boldsymbol{F}$ is found by linearization of the error model

$$
\begin{aligned}
\dot{\boldsymbol{\epsilon}} & =\boldsymbol{\Psi}_{R}^{-1}(\operatorname{ad}(\boldsymbol{\epsilon})) \boldsymbol{\omega}_{e b}^{b} \\
\dot{\boldsymbol{\omega}}_{e b}^{b} & =\boldsymbol{f}_{\omega e}(\boldsymbol{\epsilon})
\end{aligned}
$$


The state propagation matrix $\boldsymbol{F}_{k}$ is derived from the error dynamics. Given the error state vector $\boldsymbol{x}_{e}=\left[\boldsymbol{\epsilon}, \boldsymbol{\omega}_{e b}^{b}\right]^{\mathrm{T}}$ where $\mathbb{E}\{\boldsymbol{\epsilon}\}=\mathbf{0}$ and $\boldsymbol{\Psi}_{r}^{-1}(\boldsymbol{\epsilon})=\boldsymbol{I}$, which yield the discrete error dynamics

$$
\boldsymbol{x}_{e, k+1 \mid k}=\left[\begin{array}{c}
\boldsymbol{\epsilon}_{k \mid k}+T \boldsymbol{\omega}_{e b, k \mid k}^{b}+\frac{1}{2} T^{2} \boldsymbol{f}_{\omega e}\left(\boldsymbol{x}_{k \mid k}\right) \\
\boldsymbol{\omega}_{e b, k \mid k}^{b}+T \boldsymbol{f}_{\omega e}\left(\boldsymbol{x}_{k \mid k}\right)
\end{array}\right]
$$

then the linearized error equations are found as

$$
\boldsymbol{F}=\left.\frac{\partial \boldsymbol{f}\left(\boldsymbol{x}_{e}\right)}{\partial \boldsymbol{x}_{e}}\right|_{\boldsymbol{x}_{e}=\mathbf{0}}=\left[\begin{array}{cc}
\boldsymbol{I} & T \boldsymbol{I} \\
\mathbf{0} & \boldsymbol{I}
\end{array}\right]
$$

if $\boldsymbol{f}_{\omega e}=\mathbf{0}$, that is when the system is driven by Gaussian noise only.

\section{Points, Lines and Planes}

\subsection{Homogeneous Points}

Consider the homogeneous point

$$
\tilde{\boldsymbol{x}}=x_{0} \boldsymbol{e}_{0}+x_{1} \boldsymbol{e}_{1}+x_{2} \boldsymbol{e}_{2}+x_{3} \boldsymbol{e}_{3}
$$

in the 3 dimensional projective space $\mathbb{P}^{3}$. The basis of the projective space $\mathbb{P}^{3}$ is $B_{v}=\left\{\boldsymbol{e}_{0}, \boldsymbol{e}_{1}, \boldsymbol{e}_{2}, \boldsymbol{e}_{3}\right\}$, where the basis elements are orthogonal and satisfies $\boldsymbol{e}_{i} \cdot \boldsymbol{e}_{j}=\delta_{i j}$, where $\delta_{i j}$ is unity when $i=j$, and zero otherwise. The homogeneous point $\tilde{\boldsymbol{x}}$ represents a point

$$
\boldsymbol{p}=x \boldsymbol{e}_{1}+y \boldsymbol{e}_{2}+z \boldsymbol{e}_{3}
$$

in the Euclidean space $\mathbb{R}^{3}$ where $x=x_{1} / x_{0}, y=x_{2} / x_{0}$ and $z=x_{3} / x_{0}$ (Semple and Kneebone, 1952; Pottmann and Wallner, 2001). It is seen that $\tilde{\boldsymbol{x}}$ represents the Euclidean point $\boldsymbol{p}=\boldsymbol{x} / x_{0}$, where $\boldsymbol{x}=x_{1} \boldsymbol{e}_{1}+x_{2} \boldsymbol{e}_{2}+x_{3} \boldsymbol{e}_{3}$. It follows that the homogeneous point $\alpha \tilde{\boldsymbol{x}}, \alpha \neq 0$ will represent the same Euclidean point as $\tilde{\boldsymbol{x}}$. The homogeneous points $\tilde{\boldsymbol{x}}$ and $\alpha \tilde{\boldsymbol{x}}$ are therefore said to be equivalent for nonzero $\alpha$.

Concerning notation, the homogeneous point $\tilde{\boldsymbol{x}}$ will also be written in terms of the scalar $x_{0}$ and the vector $\boldsymbol{x}$ in the form $\tilde{\boldsymbol{x}}=\left(x_{0}, \boldsymbol{x}\right)$, which is used in (Pottmann and Wallner, 2001). In addition, the homogeneous point $\tilde{\boldsymbol{x}}$ will be written as the column vector $\tilde{\boldsymbol{x}}=\left[x_{1}, x_{2}, x_{3}, x_{0}\right]^{\mathrm{T}}$, which is the notation that is used in robotics and vision literature (Hartley and Zisserman, 2004; Siciliano et al., 2008).

\subsection{The Exterior Product}

The exterior product and the concept of duality is used in (Pottmann and Wallner, 2001) to define lines and planes in terms of Plücker coordinates. The exterior product on $\mathbb{P}$ is defined in terms of the basis vectors by

$$
e_{i} \wedge e_{j}=-e_{j} \wedge e_{i}=e_{i j}
$$

where $\boldsymbol{e}_{i j}$ is called a blade of grade 2, while a vector $\boldsymbol{e}_{i}$ is a blade of grade 1. It is seen that $\boldsymbol{e}_{i} \wedge \boldsymbol{e}_{i}=\boldsymbol{e}_{i i}=0$. The exterior product of two vectors $\tilde{\boldsymbol{x}}=\sum_{i=0}^{3} x_{i} \boldsymbol{e}_{i}$ and $\tilde{\boldsymbol{y}}=\sum_{i=0}^{3} y_{i} \boldsymbol{e}_{i}$ in $\mathbb{P}^{3}$ is given by

$$
\tilde{\boldsymbol{x}} \wedge \tilde{\boldsymbol{y}}=\left(\sum_{i=0}^{3} x_{i} \boldsymbol{e}_{i}\right) \wedge\left(\sum_{i=0}^{3} y_{i} \boldsymbol{e}_{i}\right)
$$

Here there are 16 different combinations of $i$ and $j$ in the $\boldsymbol{e}_{i j}$ blades. From $\boldsymbol{e}_{i i}=0$ and $\boldsymbol{e}_{i j}=-\boldsymbol{e}_{j i}$, it follows that only 6 of these are independent, and it is found that the exterior product can be written in terms of the 6 basis elements in

$$
B_{2}=\left\{\boldsymbol{e}_{01}, \boldsymbol{e}_{02}, \boldsymbol{e}_{03}, \boldsymbol{e}_{23}, \boldsymbol{e}_{31}, \boldsymbol{e}_{12}\right\}
$$

It follows that

$$
\tilde{\boldsymbol{x}} \wedge \tilde{\boldsymbol{y}}=\sum_{\boldsymbol{e}_{i j} \in B_{2}} l_{i j} \boldsymbol{e}_{i j}
$$

where

$$
l_{i j}=x_{i} y_{j}-x_{j} y_{i}
$$

The exterior product is extended to 3 factors by the associative law $\left(\boldsymbol{e}_{i} \wedge \boldsymbol{e}_{j}\right) \wedge \boldsymbol{e}_{k}=\boldsymbol{e}_{i} \wedge\left(\boldsymbol{e}_{j} \wedge \boldsymbol{e}_{k}\right)=\boldsymbol{e}_{i} \wedge \boldsymbol{e}_{j} \wedge \boldsymbol{e}_{k}=$ $\boldsymbol{e}_{i j k}$, where $\boldsymbol{e}_{i j k}$ is a blade of grade 3 . It follows that $\boldsymbol{e}_{i j k}$ is unchanged under cyclic permutations of the indices, while it changes sign under noncyclic permutations. It is noted that $\boldsymbol{e}_{i j k}$ is zero if it has a repeated index. It follows that the exterior product of 3 homogeneous vectors can be expressed in terms of the 4 basis vectors of

$$
B_{3}=\left\{\boldsymbol{e}_{123}, \boldsymbol{e}_{023}, \boldsymbol{e}_{031}, \boldsymbol{e}_{012}\right\}
$$

Therefore the exterior product of the three points $\tilde{\boldsymbol{x}}, \tilde{\boldsymbol{y}}$ and $\tilde{\boldsymbol{z}}$ will be

$$
\tilde{\boldsymbol{x}} \wedge \tilde{\boldsymbol{y}} \wedge \tilde{\boldsymbol{z}}=\sum_{\boldsymbol{e}_{i j k} \in B_{3}} m_{i j k} \boldsymbol{e}_{i j k}
$$

where $\tilde{\boldsymbol{z}}=\sum_{i=0}^{3} z_{i} \boldsymbol{e}_{i}$. Expressions for $m_{i j k}$ will be presented in the following. First, it is useful to note that the exterior product of 4 vectors will be expressed in terms of the basis $B_{4}=e_{0123}=e_{3210}$.

The geometric product of two basis vectors is written as a juxtaposition of the vectors, and is defined by (Dorst et al., 2007)

$$
\boldsymbol{e}_{i} \boldsymbol{e}_{j}=\boldsymbol{e}_{i} \cdot \boldsymbol{e}_{j}+\boldsymbol{e}_{i} \wedge \boldsymbol{e}_{j}= \begin{cases}1, & i=j \\ \boldsymbol{e}_{i j}, & i \neq j\end{cases}
$$

It follows that $\boldsymbol{e}_{i} \boldsymbol{e}_{j}=-\boldsymbol{e}_{j} \boldsymbol{e}_{i}$ whenever $i \neq j$. The inner product of two exterior products $\boldsymbol{e}_{i_{1} \ldots i_{n}}$ and $\boldsymbol{e}_{j_{1} \ldots j_{m}}$ where $m>n$ can then be expressed in terms of the geometric product as

$$
\boldsymbol{e}_{i_{1} \ldots i_{n}} \cdot \boldsymbol{e}_{j_{1} \ldots j_{m}}=\left\langle\boldsymbol{e}_{i_{1}} \ldots \boldsymbol{e}_{i_{n}} \boldsymbol{e}_{j_{1}} \ldots \boldsymbol{e}_{j_{m}}\right\rangle_{m-n}
$$


where $\langle\cdot\rangle_{k}$ denotes the terms of grade $k$. Then this can be evaluated by rearranging the terms to simplify the expression using $\boldsymbol{e}_{i} \boldsymbol{e}_{i}=1$. This is done until there are no repeated indices. It follows that the inner product of the elements of $B_{3}$ with $\boldsymbol{e}_{3210}$ are found to be

$$
\begin{aligned}
& e_{123} \cdot e_{3210}=e_{1} e_{2} e_{3} e_{3} e_{2} e_{1} e_{0}=e_{0} \\
& e_{023} \cdot e_{3210}=-e_{0} e_{2} e_{3} e_{3} e_{2} e_{0} e_{1}=-e_{1} \\
& e_{031} \cdot e_{3210}=-e_{0} e_{3} e_{1} e_{1} e_{3} e_{0} e_{2}=-e_{2} \\
& e_{012} \cdot e_{3210}=-e_{0} e_{1} e_{2} e_{2} e_{1} e_{0} e_{3}=-e_{3}
\end{aligned}
$$

The resulting vectors are the elements of the dual basis

$$
B_{3}^{*}=\left\{\boldsymbol{e}_{0},-e_{1},-e_{2},-e_{3}\right\}
$$

In the same way it is found that the dual basis of $B_{2}$ is

$$
B_{2}^{*}=\left\{\boldsymbol{e}_{23}, \boldsymbol{e}_{31}, \boldsymbol{e}_{12}, \boldsymbol{e}_{01}, \boldsymbol{e}_{02}, \boldsymbol{e}_{03}\right\}
$$

\subsection{Lines in Plücker Coordinates}

Consider a line $\mathrm{L}$ through the two Euclidean points $\boldsymbol{q}$ and $\boldsymbol{p}$ with homogeneous representations $\tilde{\boldsymbol{x}}=\left(x_{0}, \boldsymbol{x}\right)$ and $\tilde{\boldsymbol{y}}=$ $\left(y_{0}, \boldsymbol{y}\right)$ where $\boldsymbol{q}=\boldsymbol{x} / x_{0}$ and $\boldsymbol{p}=\boldsymbol{y} / y_{0}$. Then the line can be represented by the exterior product

$$
\mathrm{L}=\tilde{\boldsymbol{x}} \wedge \tilde{\boldsymbol{y}}=\sum_{\boldsymbol{e}_{i j} \in B_{2}} l_{i j} \boldsymbol{e}_{i j}
$$

where $l_{i j}$ are the Plücker coordinates of the line (Pottmann and Wallner, 2001). This can be rewritten as $\mathrm{L}=\left(\boldsymbol{l}, \boldsymbol{l}^{\prime}\right)$, where $\mathrm{L}$ is given by the pair of vectors $\boldsymbol{l}=\left[l_{01}, l_{02}, l_{03}\right]^{\mathrm{T}}$ and $\boldsymbol{l}^{\prime}=\left[l_{23}, l_{31}, l_{12}\right]^{\mathrm{T}}$. From (90) it is found that

$$
\mathrm{L}=\left(\boldsymbol{l}, \boldsymbol{l}^{\prime}\right)=\left(x_{0} \boldsymbol{y}-y_{0} \boldsymbol{x}, \boldsymbol{x} \times \boldsymbol{y}\right)
$$

and it follows that $\boldsymbol{l} \cdot \boldsymbol{l}^{\prime}=0$.

The geometric interpretation of $L$ is found by considering the direction vector $\boldsymbol{a}=\boldsymbol{p}-\boldsymbol{q}$ and the moment $\boldsymbol{q} \times \boldsymbol{a}$ of the line. It is seen that

$$
\begin{gathered}
\boldsymbol{a}=\frac{\boldsymbol{y}}{y_{0}}-\frac{\boldsymbol{x}}{x_{0}}=\frac{x_{0} \boldsymbol{y}-y_{0} \boldsymbol{x}}{x_{0} y_{0}} \\
\boldsymbol{q} \times \boldsymbol{a}=\frac{\boldsymbol{x}}{x_{0}} \times \frac{x_{0} \boldsymbol{y}-y_{0} \boldsymbol{x}}{x_{0} y_{0}}=\frac{\boldsymbol{x} \times \boldsymbol{y}}{x_{0} y_{0}}
\end{gathered}
$$

which gives $\mathrm{L}=\left(1 / x_{0} y_{0}\right)(\boldsymbol{a}, \boldsymbol{q} \times \boldsymbol{a})$. As the representation is homogeneous, an equivalent expression is

$$
\mathrm{L}=\left(\boldsymbol{l}, \boldsymbol{l}^{\prime}\right)=(\boldsymbol{a}, \boldsymbol{q} \times \boldsymbol{a})
$$

where the line is given by its direction vector $\boldsymbol{a}$ and its moment $\boldsymbol{q} \times \boldsymbol{a}$. Usually the direction vector $\boldsymbol{a}$ is scaled to be a unit vector. The point on the line that is closest to the origin is found from $\boldsymbol{q}_{\perp}=\boldsymbol{l} \times \boldsymbol{l}^{\prime}$.

It is noted that two lines $\left(\boldsymbol{l}^{\prime}, \boldsymbol{l}\right)$ and $\left(\boldsymbol{m}, \boldsymbol{m}^{\prime}\right)$ will intersect if and only if $\left(\boldsymbol{m}, \boldsymbol{m}^{\prime}\right) \cdot\left(\boldsymbol{l}^{\prime}, \boldsymbol{l}\right)=\left(\boldsymbol{m} \cdot \boldsymbol{l}^{\prime}+\boldsymbol{m}^{\prime} \cdot \boldsymbol{l}\right)=0$.

\subsection{Planes in Plücker coordinates}

A plane can be defined as the exterior product of three points $\tilde{\boldsymbol{x}}=\left(x_{0}, \boldsymbol{x}\right), \tilde{\boldsymbol{y}}=\left(y_{0}, \boldsymbol{y}\right)$ and $\tilde{\boldsymbol{z}}=\left(z_{0}, \boldsymbol{z}\right)$ as

$$
\boldsymbol{U}=\tilde{\boldsymbol{z}} \wedge \tilde{\boldsymbol{x}} \wedge \tilde{\boldsymbol{y}}=\tilde{\boldsymbol{z}} \wedge \mathrm{L}
$$

where $L=\tilde{\boldsymbol{x}} \wedge \tilde{\boldsymbol{y}}$ is the line through $\tilde{\boldsymbol{x}}$ and $\tilde{\boldsymbol{y}}$. This can be evaluated as

$$
\begin{aligned}
\boldsymbol{U} & =\left(\sum_{i=0}^{3} z_{i} \boldsymbol{e}_{i}\right) \wedge\left(\sum_{\boldsymbol{e}_{i j} \in B_{2}} l_{i j} \boldsymbol{e}_{i j}\right) \\
& =u_{0} \boldsymbol{e}_{123}-\left(u_{1} \boldsymbol{e}_{023}+u_{2} \boldsymbol{e}_{031}+u_{3} \boldsymbol{e}_{012}\right)
\end{aligned}
$$

where the coordinates are found by direct computation, or as in (Pottmann and Wallner, 2001), to be given by

$$
u_{0}=\boldsymbol{z} \cdot \boldsymbol{l}^{\prime}, \quad \boldsymbol{u}=-z_{0} \boldsymbol{l}^{\prime}+\boldsymbol{z} \times \boldsymbol{l}
$$

where $\boldsymbol{u}=\left[u_{1}, u_{2}, u_{3}\right]^{\mathrm{T}}$. The dual representation of the plane is

$$
\tilde{\boldsymbol{u}}=\boldsymbol{U} \cdot \boldsymbol{e}_{3210}=\sum_{i=0}^{3} u_{i} \boldsymbol{e}_{i}=\left(u_{0}, \boldsymbol{u}\right)
$$

A point $\tilde{\boldsymbol{w}}=\left(w_{0}, \boldsymbol{w}\right)$ will be in the plane if $\tilde{\boldsymbol{w}} \wedge \boldsymbol{U}=0$, which is equivalent to the well-known condition (Hartley and Zisserman, 2004)

$$
\tilde{\boldsymbol{w}} \cdot \tilde{\boldsymbol{u}}=0
$$

A geometric interpretation of $\tilde{\boldsymbol{u}}$ is found by defining the Euclidean point $\boldsymbol{r}=\boldsymbol{z} / z_{0}$, and the vector $\boldsymbol{b}=\boldsymbol{r}-\boldsymbol{q}$, which is in the plane. Then from (105) and (108) it is straightforward to verify that $\boldsymbol{u}=z_{0} \boldsymbol{b} \times \boldsymbol{a}$, which is normal to the plane. Moreover, it is found that $u_{0}=-\boldsymbol{q} \cdot \boldsymbol{u}$, which is proportional to the distance $\delta=\boldsymbol{q} \cdot \boldsymbol{u} /|\boldsymbol{u}|$ from the origin to the plane in the direction of $\boldsymbol{u}$.

\subsection{Lines as the intersection of planes}

Consider the two planes $\boldsymbol{U}=\tilde{\boldsymbol{z}} \wedge \mathrm{L}$ and $\boldsymbol{V}=\tilde{\boldsymbol{w}} \wedge \mathrm{L}$ defined by the common line $L$ and the points $\tilde{\boldsymbol{z}}$ and $\tilde{\boldsymbol{w}}$ where $\tilde{\boldsymbol{z}} \neq \tilde{\boldsymbol{w}}$. The corresponding dual representations are $\tilde{\boldsymbol{u}}=\boldsymbol{U} \cdot \boldsymbol{e}_{3210}=$ $\left(u_{0}, \boldsymbol{u}\right)$ and $\tilde{\boldsymbol{v}}=\boldsymbol{V} \cdot \boldsymbol{e}_{3210}=\left(v_{0}, \boldsymbol{v}\right)$. The line $\mathrm{L}$ is in both planes, and it follows that the planes intersect at $\mathrm{L}$.

The exterior product of the dual planes $\tilde{\boldsymbol{u}}$ and $\tilde{\boldsymbol{v}}$ can be found by a lengthy direct calculation to be

$$
\tilde{\boldsymbol{u}} \wedge \tilde{\boldsymbol{v}}=\alpha \mathrm{L}^{*}
$$

where $\alpha$ is a scalar and $\mathrm{L}^{*}=\mathrm{L} \cdot \boldsymbol{e}_{0123}=\left(\boldsymbol{l}^{\prime}, \boldsymbol{l}\right)$ is the dual form of the line $L=\left(\boldsymbol{l}, \boldsymbol{l}^{\prime}\right)$. The dual line is homogeneous, and the scaling can be selected so that

$$
\mathrm{L}^{*}=\tilde{\boldsymbol{u}} \wedge \tilde{\boldsymbol{v}}=\left(u_{0} \boldsymbol{v}-v_{0} \boldsymbol{u}, \boldsymbol{u} \times \boldsymbol{v}\right)
$$


The line is then found from the dual line to be

$$
\mathrm{L}=\left(\boldsymbol{u} \times \boldsymbol{v}, u_{0} \boldsymbol{v}-v_{0} \boldsymbol{u}\right)
$$

The scalar in equation (112) is given by $\alpha=-(\tilde{\boldsymbol{z}} \wedge \tilde{\boldsymbol{w}})$. $\mathrm{L}^{*}$. Let $\left(\boldsymbol{m}, \boldsymbol{m}^{\prime}\right)=\tilde{\boldsymbol{z}} \wedge \tilde{\boldsymbol{w}}$ be the line through the points $\tilde{\boldsymbol{z}}$ and $\tilde{\boldsymbol{w}}$. Then $\alpha=-\left(\boldsymbol{m}, \boldsymbol{m}^{\prime}\right) \cdot\left(\boldsymbol{l}^{\prime}, \boldsymbol{l}\right)=-\left(\boldsymbol{m} \cdot \boldsymbol{l}^{\prime}+\boldsymbol{m}^{\prime} \cdot \boldsymbol{l}\right)$, which is nonzero whenever $\left(\boldsymbol{m}, \boldsymbol{m}^{\prime}\right)$ and $\left(\boldsymbol{l}^{\prime}, \boldsymbol{l}\right)$ does not intersect.

\section{Vision}

\subsection{Camera model}

The camera model is given by (Ma et al., 2003; Hartley and Zisserman, 2004)

$$
\begin{aligned}
\lambda \boldsymbol{s} & =\left[\boldsymbol{R}_{c} \mid \boldsymbol{t}_{c}\right] \tilde{\boldsymbol{r}} \\
\boldsymbol{p} & =\boldsymbol{K} \boldsymbol{s}
\end{aligned}
$$

where $s=\left[s_{x}, s_{y}, 1\right]^{\mathrm{T}}$ is the homogeneous vector of normalized image coordinates, $\boldsymbol{p}=\left[p_{x}, p_{y}, 1\right]^{\mathrm{T}}$ is the homogeneous vector of pixel coordinates, $\boldsymbol{P}=\left[\boldsymbol{R}_{c} \mid \boldsymbol{t}_{c}\right]$ is the uncalibrated camera model, where $\boldsymbol{R}_{c}$ and $\boldsymbol{t}_{c}$ define the homogeneous transformation matrix

$$
\boldsymbol{T}_{c}=\left[\begin{array}{cc}
\boldsymbol{R}_{c} & \boldsymbol{t}_{c} \\
\mathbf{0}^{\mathrm{T}} & 1
\end{array}\right]
$$

from the camera frame to the object frame. The homogeneous vector $\tilde{\boldsymbol{r}}=\left[\boldsymbol{r}^{\mathrm{T}}, 1\right]^{\mathrm{T}}$ gives the position of a world point in the coordinates of the object frame, $\lambda>0$ is the depth parameter and

$$
\boldsymbol{K}=\left[\begin{array}{ccc}
f_{x} & 0 & c_{x} \\
0 & f_{y} & c_{y} \\
0 & 0 & 1
\end{array}\right]
$$

is the intrinsic camera calibration matrix.

\subsection{Determination of a line from stereo images}

Consider two cameras used in a stereo arrangement with uncalibrated camera models $\boldsymbol{P}_{1}=[\boldsymbol{I} \mid \mathbf{0}]$ and $\boldsymbol{P}_{2}=[\boldsymbol{R} \mid \boldsymbol{t}]$. This means that the problem is described in the coordinates of camera frame 1, and that the displacement from camera frame 2 to camera frame 1 is given by the rotation matrix $\boldsymbol{R}$ and the translation vector $\boldsymbol{t}$. A homogeneous world point $\tilde{\boldsymbol{r}}=\left[\boldsymbol{r}^{\mathrm{T}}, 1\right]^{\mathrm{T}}$ is then mapped to the homogeneous normalized coordinate vectors

$$
\lambda_{1} \boldsymbol{s}_{1}=\boldsymbol{P}_{1} \tilde{\boldsymbol{r}}=\boldsymbol{r}, \quad \lambda_{2} \boldsymbol{s}_{2}=\boldsymbol{P}_{2} \tilde{\boldsymbol{r}}=\boldsymbol{R} \boldsymbol{r}+\boldsymbol{t}
$$

Suppose that the world point $\tilde{\boldsymbol{r}}$ is an arbitrary point on the line $L$ in 3 dimensional Euclidean space. Then the point $\tilde{\boldsymbol{r}}$ is mapped to a point $\boldsymbol{s}_{1}=\lambda_{1}^{-1} \boldsymbol{P}_{1} \tilde{\boldsymbol{r}}$ on a line $\boldsymbol{\ell}_{1}$ in image plane 1 , which means that $\boldsymbol{\ell}_{1}^{\mathrm{T}} \boldsymbol{s}_{1}=\boldsymbol{\ell}_{1}^{\mathrm{T}} \boldsymbol{P}_{1} \tilde{\boldsymbol{r}}=0$. This is true for any point $\tilde{\boldsymbol{r}}$ on the plane $\tilde{\boldsymbol{u}}$ through the origin of camera 1 and the line $L$. This means that this plane is given by

$$
\tilde{\boldsymbol{u}}=\boldsymbol{P}_{1}^{\mathrm{T}} \boldsymbol{\ell}_{1}=\left[\begin{array}{c}
\boldsymbol{I} \\
\mathbf{0}^{\mathrm{T}}
\end{array}\right] \boldsymbol{\ell}_{1}=\left[\begin{array}{c}
\boldsymbol{\ell}_{1} \\
0
\end{array}\right],
$$

In the same way, it follows from $\boldsymbol{\ell}_{2}^{\mathrm{T}} \boldsymbol{s}_{2}=\boldsymbol{\ell}_{2}^{\mathrm{T}} \boldsymbol{P}_{2} \tilde{\boldsymbol{r}}=0$ that the plane $\tilde{\boldsymbol{v}}$ trough the origin of camera 2 and the line $L$ must be given by

$$
\tilde{\boldsymbol{v}}=\boldsymbol{P}_{2}^{\mathrm{T}} \boldsymbol{\ell}_{2}=\left[\begin{array}{c}
\boldsymbol{R}^{\mathrm{T}} \\
\boldsymbol{t}^{\mathrm{T}}
\end{array}\right] \boldsymbol{\ell}_{2}=\left[\begin{array}{c}
\boldsymbol{R}^{\mathrm{T}} \boldsymbol{\ell}_{2} \\
\boldsymbol{t}^{\mathrm{T}} \boldsymbol{\ell}_{2}
\end{array}\right]
$$

In Plücker coordinate form this is written

$$
\tilde{\boldsymbol{u}}=\left(0, \boldsymbol{\ell}_{1}\right), \quad \tilde{\boldsymbol{v}}=\left(\boldsymbol{t}^{\mathrm{T}} \boldsymbol{\ell}_{2}, \boldsymbol{R}^{\mathrm{T}} \boldsymbol{\ell}_{2}\right)
$$

This means that if $\boldsymbol{R}$ and $\boldsymbol{t}$ are known, and the lines $\boldsymbol{\ell}_{1}$ and $\boldsymbol{\ell}_{2}$ can be determined, the planes $\tilde{\boldsymbol{u}}$ and $\tilde{\boldsymbol{v}}$ can be computed, and the dual of the line $L$ can be computed from

$$
\mathrm{L}^{*}=\tilde{\boldsymbol{u}} \wedge \tilde{\boldsymbol{v}}
$$

Then the line is found from (113) to be

$$
\mathrm{L}=\left(\boldsymbol{\ell}_{1} \times\left(\boldsymbol{R}^{\mathrm{T}} \boldsymbol{\ell}_{2}\right),-\left(\boldsymbol{t}^{\mathrm{T}} \boldsymbol{\ell}_{2}\right) \boldsymbol{\ell}_{1}\right) .
$$

The measured element, denoted as $Z$ in the filter and which is an element of $S O(3)$, is found by computing the angle between the reference line and the measured line, and finding the orthogonal unit vector on these lines. The measured rotation matrix is then generated by using Rodrigues formula as given in Equation (63). For the special case when the measured line is equal to the reference line, the measured matrix is defined as the identity matrix.

\subsection{Line detection in the image plane}

The applied line detector is a pipeline-implementation based on the Canny edge detector and the Hough transformation for lines, and it is used to obtain the representation of the line projected onto each image plane in the multiview configuration. The Canny edge detector is a multistage algorithm which extracts structural information from provided camera readings, such as edges and corners, and returns a binary image containing elements representing edges or corners. The binary image is provided as input for the Hough transformation, which is applied to find a set of lines which are most likely to be actual lines in an image. A line can be written as the function

$$
\rho=x \cos \theta+y \sin \theta
$$

which are the line coordinates given by $\rho$, which is the shortest distance between the origin and the line, and $\theta$, 


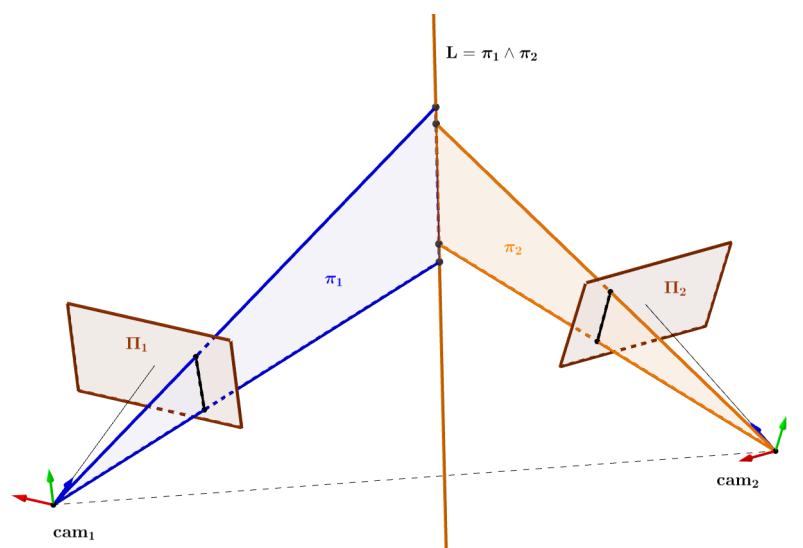

Figure 3: Two planes, $\boldsymbol{\pi}_{1}$ and $\boldsymbol{\pi}_{2}$, are generated though the origin of each camera coordinate system and the detected line on each image plane, and the intersection generates the line, $\mathrm{L}$, representing the detected wire in $3 \mathrm{D}$ space.

which is the angle between the $x$ axis and the normal vector of the line. A voting routine is applied, and the lines which are most likely to exist in the image are stored in the memory. This is done by using the Hough transformation for lines (Duda and Hart, 1972).

The line detecting pipeline returns a set of lines $\left(\theta_{i}, \rho_{i}\right)$ in the pixel plane, which corresponds to the line $\boldsymbol{\lambda}=$ $[\cos \theta, \sin \theta,-\rho]^{\mathrm{T}}$ in pixel coordinates. The line is converted to normalized image coordinates by the transformation

$$
\ell=\boldsymbol{K}^{\mathrm{T}} \boldsymbol{\lambda}
$$

which results from homography $s=K^{-1} p$, which is the inverse of (115).

\section{Spherical Pendulum Dynamics}

The inertial frame $i$ is defined with the $z$ axis vertically upwards, parallel with the line when the wire is at rest. The body-fixed frame is denoted $b$ with the $z$ axis parallel with the line describing the wire at time instance $t_{k}$. The rotation matrix from the inertial frame to the body frame is written $\boldsymbol{R}=\boldsymbol{R}_{y}(\theta) \boldsymbol{R}_{x}(\phi)$ where $\phi$ is the roll angle and $\theta$ is the pitch angle.

The model describing the dynamics of the pendulum can be described by the Euler equations of motion in the body frame $b$ as

$$
\boldsymbol{J} \dot{\boldsymbol{\omega}}^{b}+\boldsymbol{\omega}^{b} \times \boldsymbol{J} \boldsymbol{\omega}^{b}=\boldsymbol{\rho}^{b} \times \boldsymbol{g}^{b}
$$

where $\boldsymbol{J}$ is the inertia matrix describing the mass properties of the payload, $\boldsymbol{\rho}^{b}=-L \boldsymbol{z}^{b}$ describes the center of mass of the payload relative to the pivot point as a vector of length $L$ in the opposite direction of $\boldsymbol{z}^{b}$. The vector $\boldsymbol{g}^{b}=m g \boldsymbol{R}_{i}^{b} \boldsymbol{z}^{i}$

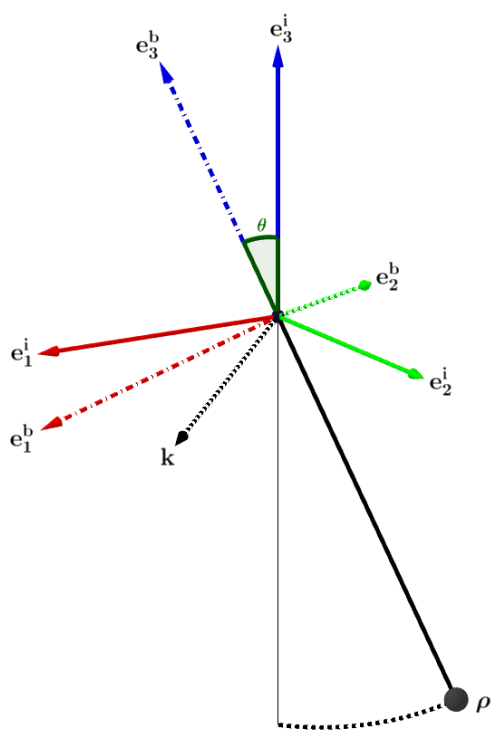

Figure 4: Let the third principal axis of the body frame, $\boldsymbol{e}_{3}^{b}$, be co-linear with the direction of the observed line. The stable attitude is defined as the line with direction vector that is co-linear with the third principal axis of the inertial frame, $e_{3}^{i}$. The composite rotation can be expressed as a rotation about a single axis, $\boldsymbol{k}$, as $\boldsymbol{R}_{b}^{i}=\exp \left(\theta \boldsymbol{k}^{\times}\right)$.

describes the force of gravity, where $m$ denotes the mass of the payload, and $g$ is the gravitational acceleration. Hence, the product $\boldsymbol{\rho}^{b} \times \boldsymbol{g}^{b}$ describes the torque generated by the gravitational force. Note that the yaw parameter (rotation about the third principal axis) is not observable in the given case since the payload is considered to be a point mass, and a rotation about this axis is not observable in the given case.

We assume that the mass of the payload is constant, and that the $b$ frame is fixed to the body $b$, which implies that the inertia matrix is constant. Furthermore, we assume that the origin of $b$ is located in the pivot point. The center of mass of the payload, given in the $b$ frame, is denoted

$$
\boldsymbol{\rho}=L \cdot[0,0,-1]^{\mathrm{T}}
$$

as illustrated in Figure 4. We assume an inertia matrix on the form

$$
\boldsymbol{J}=\left[\begin{array}{ccc}
J_{t} & 0 & 0 \\
0 & J_{t} & 0 \\
0 & 0 & J_{a}
\end{array}\right]
$$

where, due to the parallel axis theorem, $J_{t}=m L^{2}$, and $J_{a}$ is assumed to be small.

By neglecting, $J_{a}$, we find the dynamics to be

$$
\dot{\boldsymbol{\omega}}^{b}=\left[\begin{array}{c}
-\omega_{y} \omega_{z} \\
\omega_{x} \omega_{z} \\
0
\end{array}\right]+\frac{g}{L} \boldsymbol{z}^{b} \times \boldsymbol{R}_{b}^{i} \boldsymbol{z}^{b}
$$


which is the same as the model presented in Shen et al. (2004). We find that, for a point mass, the acceleration about the wire axis is small, and the velocity is therefore approximately constant. We assume it to be zero since this component is not observable in the given setup.

Therefore, given the vector $\boldsymbol{x}_{e}=\left[\boldsymbol{\epsilon}^{\mathrm{T}}, \boldsymbol{\omega}^{\mathrm{T}}\right]^{\mathrm{T}}$, the linearized motion model is found as

$$
\begin{aligned}
\boldsymbol{F} & =\left.\frac{\partial f\left(\boldsymbol{x}_{e}\right)}{\partial \boldsymbol{x}_{e}}\right|_{\boldsymbol{x}_{e}=\mathbf{0}} \\
& =\frac{\partial}{\partial \boldsymbol{x}_{e}}\left[\begin{array}{cc}
\boldsymbol{\epsilon}+\Psi_{r}^{-1}(\boldsymbol{\epsilon})\left(T \boldsymbol{\omega}-\frac{1}{2} T^{2} \frac{g}{L} \boldsymbol{\epsilon}\right) \\
\boldsymbol{\omega}-T \frac{g}{L} \boldsymbol{\epsilon}
\end{array}\right] \\
& =\left[\begin{array}{cc}
\boldsymbol{I}-\frac{1}{2} T^{2} \frac{g}{L} \boldsymbol{I} & T \boldsymbol{I} \\
-T \frac{g}{L} \boldsymbol{I} & \boldsymbol{I}
\end{array}\right]
\end{aligned}
$$

since the inverted right Jacobian evaluates to identity as $\mathbb{E}\{\boldsymbol{\epsilon}\}=\mathbf{0}$.

\section{Simulations}

The EKF on $S O(3)$ was tested in a simulation study of a 3-dimensional spherical pendulum based on the model in Equation (126). The EKF was used to estimate the rotation matrix and the angular velocity. The process covariance matrix was set to $\boldsymbol{R}_{n}=\sigma_{r}^{2} \boldsymbol{I}_{6 \times 6}$ where $\sigma_{r}^{2}=0.01$ was the variance of the model. The measurement covariance matrix was set to $\boldsymbol{Q}_{w}=\sigma_{q}^{2} \boldsymbol{I}_{3 \times 3}$ where $\sigma_{q}^{2}=0.1$ was the variance of the measurements.

The plotted data will show the Euler angles roll and pitch, which are found as

$$
\begin{aligned}
& \phi=\operatorname{atan} 2\left(r_{32}, r_{33}\right) \\
& \theta=\operatorname{atan} 2\left(-r_{31}, \sqrt{r_{32}^{2}+r_{33}^{2}}\right)
\end{aligned}
$$

given a rotation matrix $\boldsymbol{R}=\left\{r_{i j}\right\}$.

The simulated case had an initial condition for the rotation matrix that was given by $\phi(0)=57.3^{\circ}$ (roll) and $\theta(0)=28.6^{\circ}$ (pitch). The initial angular velocity was given as $\boldsymbol{\omega}^{b}=[-1,1,0]^{\mathrm{T}} \frac{\mathrm{rad}}{\mathrm{s}}$. White Gaussian noise with covariance $0.025 I$ was multiplied into the simulated signal, and as seen in Figure 6, the estimated values converged within 2.5 seconds in this particular case. Figure 5 shows the estimated values and the corresponding measurements.

\section{Experiments and Results}

The EKF formulated on $S O(3)$, as described in Section (4), was used in two experiments for estimation of the pendulum motion. The dynamics given in Equation (129) was used for the state prediction, and the corresponding linearized dynamics given in Equation (130) was used to propagate the
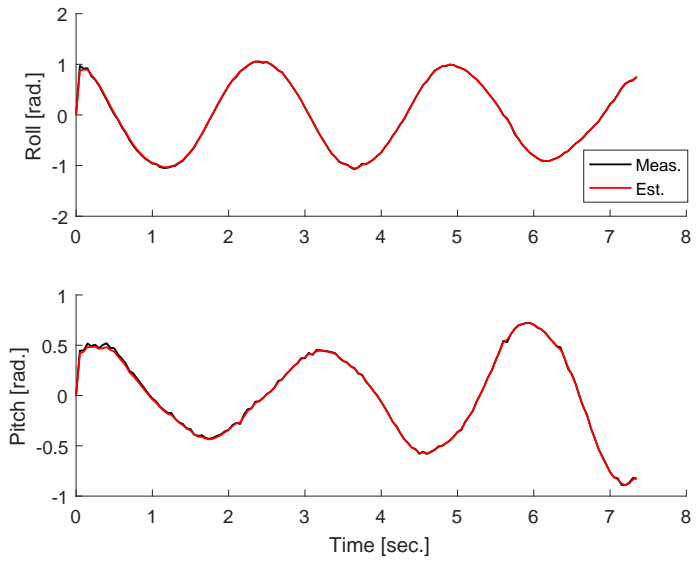

Figure 5: The system was simulated where the covariance describing the uncertainties in the measurements was 10 times larger than the covariance describing the uncertainties in the system model.

covariance matrix. The wire length of the spherical pendulum was measured to be $L=1.3 \mathrm{~m}$, and the resulting period of the linearized model was $T=2 \pi \sqrt{g / L}=2.28 \mathrm{~s}$. The system was implemented on a system running on a laptop with a $2.6 \mathrm{GHz} \mathrm{CPU}$, and the pixel resolution of the sampled pictures were $480 \times 640$. The sampling rate of the camera system was measured to be in the range $15-18 \mathrm{~Hz}$.

The first experiment was a case where the pendulum was oscillating approximately parallel to the image plane, which will be referred to as roll motion. In this case, the estimates (see Figure (7)) showed that the roll parameter oscillated with an amplitude of $0.134 \mathrm{rad}$ and the pitch parameter oscillated with an amplitude of $0.018 \mathrm{rad}$.

The second experiment was a case where the pendulum was oscillating approximately perpendicular to the image plane, which will be referred to as pitch motion. In this case, the estimates (see Figure (9)) showed that the roll oscillated with an amplitude of $0.037 \mathrm{rad}$ and the pitch oscillated with an amplitude of $0.15 \mathrm{rad}$.

The covariance matrix describing the process noise was, in both cases, set to

$$
\boldsymbol{R}_{n}=\left[\begin{array}{cc}
\boldsymbol{R}_{R} & \mathbf{0} \\
\mathbf{0} & \boldsymbol{R}_{\omega}
\end{array}\right] \in \mathbb{R}^{6 \times 6}
$$

where $\boldsymbol{R}_{n}=\boldsymbol{R}_{\omega}=10^{-4} \boldsymbol{I}_{3 \times 3}$. The covariance matrix describing measurement noise was given on the form

$$
\boldsymbol{Q}_{n}=\left[\begin{array}{ccc}
Q_{\phi \phi} & 0 & 0 \\
0 & Q_{\theta \theta} & 0 \\
0 & 0 & Q_{\psi \psi}
\end{array}\right]
$$

and the matrix $\boldsymbol{Q}_{n}=\operatorname{diag}(0.5,0.05,1)$ was used for the experiments. The parameter $Q_{\psi \psi}$ can be set arbitrarily high as the measurements of the $\psi$ angle are not observable in the studied system configuration. The parameter $Q_{\phi \phi}$ is set 

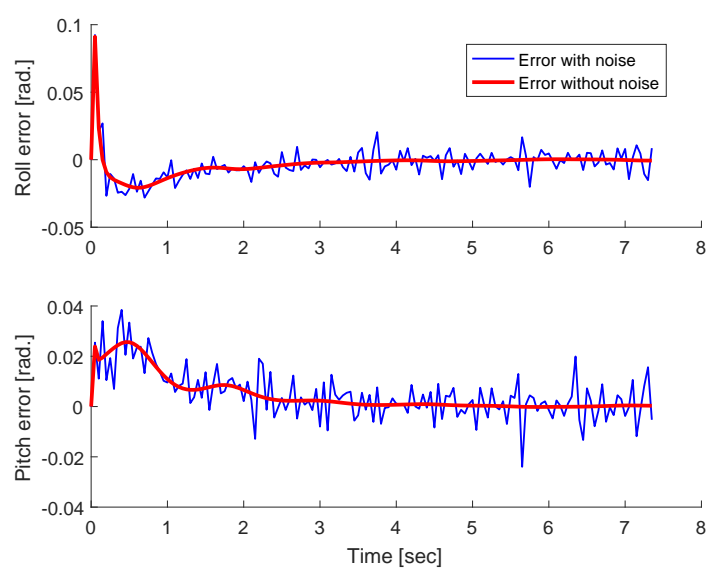

Figure 6: The initial errors were large due to the initial conditions, and the peak of the pitch value is due to a lower weight on its measurements while also being prone to a wrong initial estimate of the angular velocity. The error offsets in the noiseless case converged to zero.

to a high value as the selected image resolution results in a low resolution of the parameter describing roll motion. Roll is in this case equivalent to depth motion, which correspond to motion perpendicular to the image plane. The motion parallel to the image plane is called pitch, and the parameter describing the uncertainties of the pitch values, $Q_{\theta \theta}$, is lower than $Q_{\phi \phi}$ since the camera configuration captures such displacements better than depth motion.
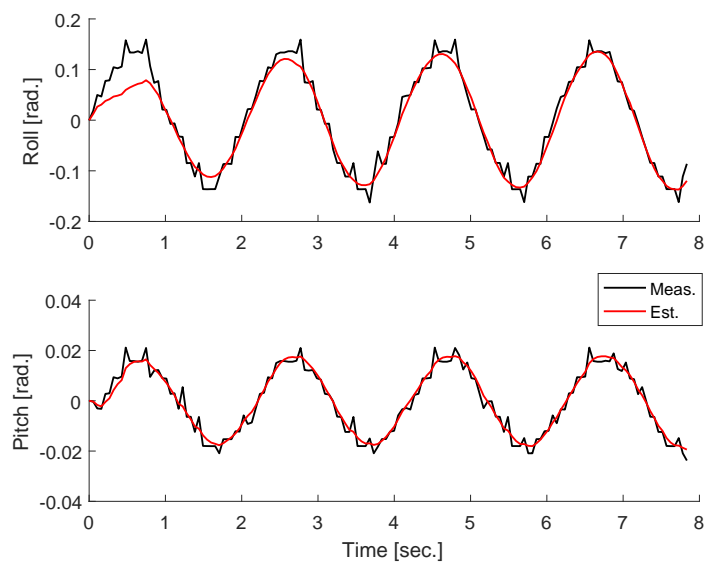

Figure 7: The measured and estimated values of the roll case.
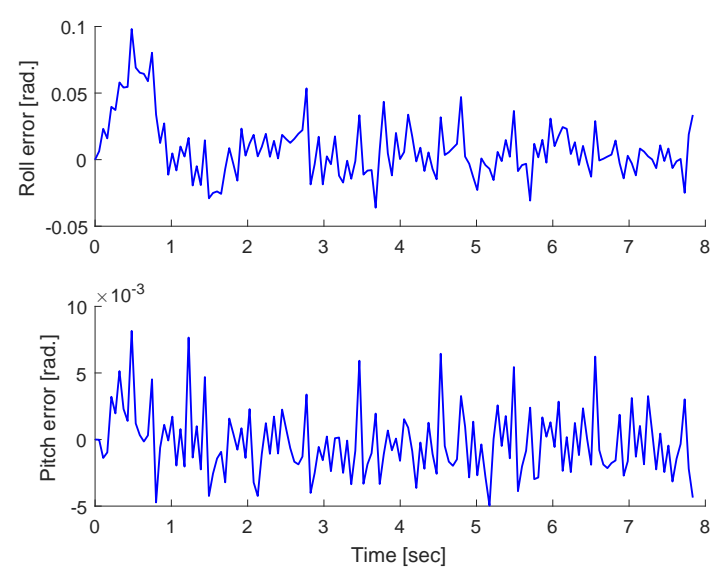

Figure 8: The error between the measurements and the estimates of the roll case.
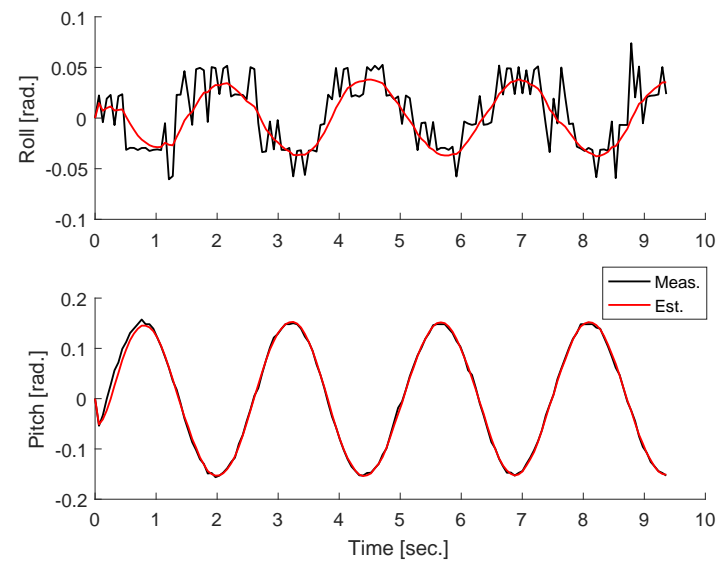

Figure 9: The measured and estimated values of the pitch case.
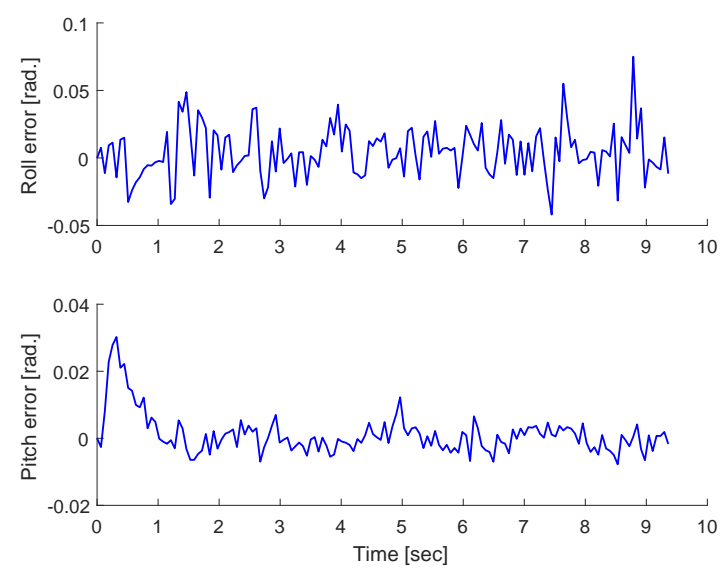

Figure 10: The error between the measurements and the estimates of the pitch case. 


\section{Discussion}

\subsection{Comparison with the D-EKF-LG}

The proposed EKF on $S O(3)$ was compared to the D-EKFLG as presented in Bourmaud et al. (2013). The filters were compared both with the experimental data sets, and the simulated data for the spherical pendulum, while using identical noise descriptions, the same numerical solver, and identical time steps. The expressions used in the D-EKF-LG filter are more involved, but since most of the additional computations evaluate close to identity matrices, the estimates of both filters are close to identical. There was no significant difference in the computed outputs of the proposed EKF on $S O(3)$ and the D-EKF-LG, as visualized in Figure (11). However, due to a simpler formulation, the computational burden of the proposed EKF was lower due to fewer computations in each iteration, leading to a faster performance. Tables 1 and 2, contain the computed root mean square errors (RMSE) on the error signal from each data set using the EKF on $S O(3)$ and the D-EKF-LG respectively.
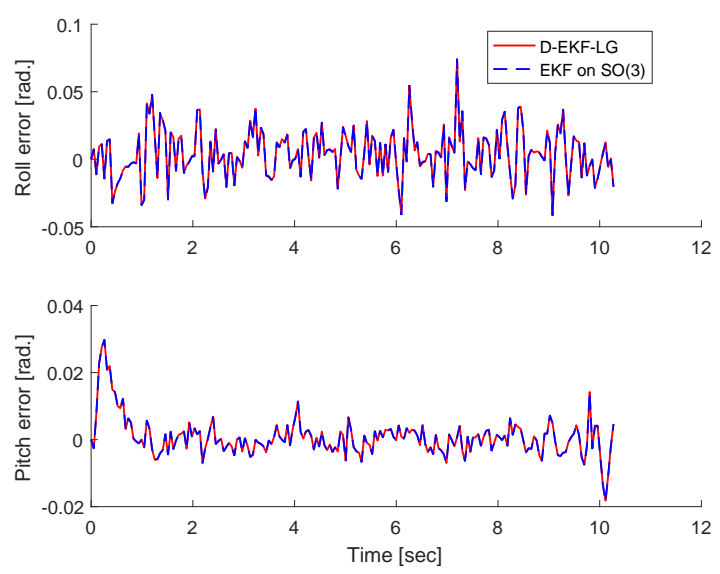

Figure 11: The D-EKF-LG and the proposed EKF on $S O(3)$ have no distinct differences when interpreting the given data sets, which in this particular case is the error plot from the pitch case from both of the estimators.

\begin{tabular}{||cccc||}
\hline RMSE & Roll case & Pitch case & Simulation \\
\hline \hline$\phi$ & 0.0187 & 0.0211 & 0.0092 \\
\hline$\theta$ & 0.0058 & 0.0024 & 0.0067 \\
\hline
\end{tabular}

Table 1: RMSE values for the experimental roll and pitch experiments, and for a simulated dataset using the EKF on $S O(3)$ formulation

\begin{tabular}{||cccc||}
\hline RMSE & Roll case & Pitch case & Simulation \\
\hline \hline$\phi$ & 0.0186 & 0.0211 & 0.0092 \\
\hline$\theta$ & 0.0058 & 0.0024 & 0.0068 \\
\hline
\end{tabular}

Table 2: RMSE values for the experimental roll and pitch experiments, and for a simulated dataset with DEKF-LG

\subsection{Modelling Aspects}

An alternative to the model used in this paper is to derive the model using generalized coordinates. The following dynamics can be obtained using the Lagrange formulation (Abdel-Rahman et al., 2003)

$$
\begin{aligned}
\ddot{\phi} \cos \theta & =2 \dot{\phi} \dot{\theta} \sin \theta-\frac{g}{L} \sin \phi \\
\ddot{\theta} & =-\dot{\phi}^{2} \sin \theta \cos \theta-\frac{g}{L} \cos \phi \sin \theta
\end{aligned}
$$

For small angles, this formulation can be linearized, and a simple Kalman filter can be used to estimate the system states as generalized coordinates. However, the nonlinearities in this formulation are introduced through the kinematics of the Euler angles, such that they appear in the EKF model. This type of nonlinearities is, however, not encountered in the EKF on Lie groups as presented in this paper.

\section{Conclusion}

A camera sensor configuration based on stereo vision was used to track a wire under tension using an edge feature detector in the vision part. The direction of the line was found by intersecting two planes generated as the plane intersecting three spatial points in each camera frame and the pendulum angles were measured using the resulting line. A new formulation for an EKF on Lie groups has been presented and applied to $S O(3)$ to estimate the angular displacement of the payload. The estimator was supported by model of the spherical pendulum which was formulated using the rotation matrix to describe the gravitational torque. The proposed EKF on $S O(3)$ is based on the kinematic differential equation of the logarithm, and the formulation is less complex and easier to implement than previously proposed estimators like the D-EKF-LG filter. At the same time the performance of the proposed EKF was shown in experiments and simulations on $S O(3)$ to be of the same accuracy as the D-EKF-LG filter.

\section{Acknowledgments}

The research presented in this paper was funded by the Norwegian Research Council under Project Number 237896, SFI Offshore Mechatronics. 


\section{References}

Abdel-Rahman, E. M., Nayfeh, A. H., and Masoud, Z. N. Dynamics and control of cranes: A review. Journal of Vibration and Control, 2003. 9:863-908. doi:10.1177/1077546303009007007.

Barfoot, T. D. and Furgale, P. T. Associating uncertainty with three-dimensional poses for use in estimation problems. IEEE Trans. Robotics, 2014. 30(3):679-693. doi:10.1109/TRO.2014.2298059.

Bisgaard, M., la Cour-Harbo, A., Johnson, E. N., and Bendtsen, J. D. Vision aided state estimator for helicopter slung load system. IFAC Proceedings Volumes, 2007. 40(7):425-430. doi:10.3182/20070625-5-FR2916.00073.

Bortz, J. E. A new mathematical formulation for strapdown inertial navigation. IEEE Trans. Aerospace and Electronic Systems, 1970. AES-7(1):61-666. doi:10.1109/TAES.1971.310252.

Bourmaud, G., Mégret, R., Arnaudon, M., and Giremus, A. Continuous-discrete extended Kalman filter on matrix Lie groups using concentrated Gaussian distributions. Journal of Mathematical Imaging and Vision, 2015. 51(1):209-228. doi:10.1007/s10851-014-0517-0.

Bourmaud, G., Mégret, R., Giremus, A., and Berthoumieu, Y. Discrete extended Kalman filter on Lie groups. 21st European Signal Processing Conf. (EUSIPCO), 2013.

Brossard, M., Bonnabel, S., and Condomines, J.-P. Unscented filtering on Lie groups. In IROS 2017, EEE/RSJ International Conference on Intelligent Robots and Systems. 2017. doi:10.1109/IROS.2017.8206066.

Bullo, F. and Murray, R. M. Proportional Derivative (PD) Control on the Euclidean Group. CDS Technical Report 95-010, California Institute of Technology, 1995.

Cesic, J., Markovic, I., Bukal, M., and Petrovic, I. Extended information filter on matrix Lie groups. Automatica, $2017 . \quad$ (82):226-234 doi:10.1016/j.automatica.2017.04.056.

Chirikjian, G. S. Stochastic Models, Information Theory, and Lie Groups, Volume 2. Birkhuser, 2012.

Crassidis, J. L. and Markley, F. L. Unscented filtering for spacecraft attitude estimation. Journal of Guidance, Control, and Dynamics, 2003. 26(4):536-542. doi: $10.2514 / 2.5102$.

Crassidis, J. L., Markley, F. L., and Cheng, Y. Survey of nonlinear attitude estimation methods. Journal of Guidance, Control, and Dynamics, 2007. 30(1):12-28. doi:10.2514/1.22452.
Crouch, P. E. and Grossman, R. Numerical integration of ordinary differential equations on manifolds. Journal of Nonlinear Science, 1993. 3(1). doi:10.1007/BF02429858.

Dorst, L., Fontijne, D., and Mann, S. Geometric Algebra for Computer Science: An Object-Oriented Approach to Geometry. Morgan Kaufmann Publishers Inc., San Francisco, CA, USA, 2007.

Duda, R. O. and Hart, P. E. Use of the Hough transformation to detect lines and curves in pictures. Communications of the ACM, 1972. 15(1):1115. doi:10.1145/361237.361242.

Faraut, J. Analysis on Lie Groups: An Introduction. Cambridge Studies in Advanced Mathematics. Cambridge University Press, 2008. doi:10.1017/CBO9780511755170.

Filipe, N., Kontitsis, M., and Tsiotras, P. Extended Kalman filter for spacecraft pose estimation using dual quaternions. Journal of Guidance, Control, and Dynamics, 2015. 38(9):1625-1641. doi:10.1109/ACC.2015.7171823.

Hall, B. C. Lie Groups, Lie Algebras, and Representations. An Elementary Introduction. Graduate Texts in Mathematics. Springer, Berlin, Heidelberg, New York, 2003.

Hartley, R. I. and Zisserman, A. Multiple View Geometry in Computer Vision. Cambridge University Press, ISBN: 0521540518, second edition, 2004.

Inukai, T. and Yoshida, Y. Control of a boom crane using installed stereo vision. 6th International Conference on Sensing Technology (ICST), 2012. doi:10.1109/ICSensT.2012.6461668.

Iserles, A., Munthe-Kaas, H., Nørsett, S., and Zanna, A. Lie-group methods. Acta Numerica, 2005. doi:10.1017/S0962492900002154.

Kim, Y.-S., Hong, K.-S., and Sul, S.-K. Anti-sway control of container cranes: Inclinometer, observer, and state feedback. International Journal of Control, Automation and Systems, 2004. 2(4):435-449.

Klarsfeld, S. and Oteo, J. A. The Baker-CampbellHausdorff formula and the convergence of the Magnus expansion. J. Phys. A: Math. Gen., 1989. 22:45654572. doi:10.1088/0305-4470/22/21/018.

Lefferts, E. J., Markley, F. L., and Shuster, M. D. Kalman filtering for spacecraft attitude estimation. Journal of Guidance, Control, and Dynamics, 1982. 5(5):417-429. doi:10.2514/3.56190. 
Loianno, G., Watterson, M., and V., K. Visual inertial odometry for quadrotors on se(3). IEEE International Conference on Robotics and Automation (ICRA), 2016. pages 1544-1551. doi:10.1109/ICRA.2016.7487292.

Ma, Y., Soatto, S., Kosecka, J., and Sastry, S. S. An Invitation to 3-D Vision: From Images to Geometric Models. SpringerVerlag, 2003.

Munthe-Kaas, H. Lie-butcher theory for runge-kutta methods. BIT Numerical Mathematics, 1995. 35(4):572-587. doi:10.1007/BF01739828.

Owren, B. and Marthinsen, A. Runge-kutta methods adapted to manifolds and based on rigid frames. BIT Numerical Mathematics, 1999. 39(1):116-142. doi:10.1023/A:1022325426017.

Park, F. Distance metrics on the rigid-body motions with applications to mechanism design. J. Mechanical Design, 1995. 117(1):48-54. doi:10.1115/1.2826116.

Pittelkau, M. E. Rotation vector in attitude estimation. Journal of Guidance, Control, and Dynamics, 2003. 26(6):855-860. doi:10.2514/2.6929.

Pottmann, H. and Wallner, J. Computational Line Geometry. Springer-Verlag, Berlin, 2001.
Rauscher, F., Nann, S., and Sawodny, O. Motion control of an overhead crane using a wireless hook mounted IMU. Annual American Control Conference (ACC), Milwaukee, USA, 2018. doi:10.23919/ACC.2018.8431170.

Semple, J. G. and Kneebone, G. T. Algebraic projective geometry. Oxford Classic Series. Clarendon Press, Oxford, 1952.

Shen, J., Sanyal, A. K., Chaturvedi, N. A., Bernstein, D., and McClamroch, H. Dynamics and control of a $3 \mathrm{~d}$ pendulum. 43rd IEEE Conference of Decision and Control, 2004. pages 323 - 328. doi:10.1109/CDC.2004.1428650.

Siciliano, B., Sciavicco, L., Villani, L., and Oriolo, G. Robotics: Modelling, Planning and Control. Springer Publishing Company, Incorporated, 1st edition, 2008.

Wang, Y. and Chirikjian, G. S. Error propagation on the Euclidean group with applications to manipulator kinematics. Trans. on Robotics, 2006. 22(4):591-602. doi:10.1109/TRO.2006.878978.

Wolfe, K. C., Mashner, M., and Chirikjian, G. S. Bayesian fusion on Lie groups. Journal of Algebraic Statistics, 2011. 2(1):75-97. doi:10.18409/jas.v2i1.11. 